\title{
Self-adjoint wave equations for dynamical perturbations of self-gravitating fields
}

\author{
O. Sarbach, M. Heusler ${ }^{\star \dagger}$, and O. Brodbeck ${ }^{\dagger}$ \\ ${ }^{\star}$ Institute for Theoretical Physics, University of Zurich, CH-8057 Zurich, Switzerland \\ Time-steps GMbH, CH-8910 Zurich, Switzerland
}

(November 1, 2018)

\begin{abstract}
It is shown that the dynamical evolution of linear perturbations on a static space-time is governed by a constrained wave equation for the extrinsic curvature tensor. The spatial part of the wave operator is manifestly elliptic and self-adjoint. In contrast to metric formulations, the curvature-based approach to gravitational perturbation theory generalizes in a natural way to self-gravitating matter fields. It is also demonstrated how to obtain symmetric pulsation equations for self-gravitating non-Abelian gauge fields, Higgs fields and perfect fluids. For vacuum fluctuations on a vacuum space-time, the Regge-Wheeler and Zerilli equations are rederived.
\end{abstract}

\section{INTRODUCTION}

Perturbation theory finds several interesting applications in general relativity. A prominent example is the close limit approximation to black hole collisions [1]. Besides providing estimates for the energy emitted by gravitational radiation, the close limit results also play an important role in testing existing numerical codes for full non-linear general relativity. Further examples comprise the linear stability analysis of neutron stars or black holes with matter fields. Recently, a perturbation approach has also been used to find new stationary solutions and to prove local uniqueness results for hairy black holes and self-gravitating solitons [2] [3] [4].

For vacuum gravity perturbation theory is highly developed, and even for self-gravitating systems important general properties of the perturbation equations were established. In particular, it has been shown that a manifestly hyperbolic, gauge-invariant formulation of gravitational perturbation theory does exist for static background configurations [5], [6]. On the other hand, it is known for a wide class of matter models that the pulsation equations governing spherical perturbations of static, spherically symmetric field configurations are manifestly symmetric and derivable from the second variation of the Komar mass [7. However, only for special systems, such as vacuum gravity and the EinsteinMaxwell system for a spherically symmetric background, formulations are known for which the perturbations are governed by a wave equation which is hyperbolic and symmetric. Symmetric formulations are most valuable, since they provide the possibility to discuss stability issues by rigorous means, applying methods from spectral theory.

The commonly adopted procedure to derive the perturbation equations for spherically symmetric black holes is to first use the symmetry of the background in order to expand the metric in terms of spherical harmonics. Then, the pulsation equations are derived for gauge-invariant quantities. For vacuum perturbations of vacuum gravity, this leads to the Regge-Wheeler equation [8] in the odd-parity sector and to the Zerilli equation [9] for evenparity perturbations. Both equations have the form of symmetric wave equations and, as an important consequence, the stability of the Schwarzschild metric within linear perturbation theory can be established. This result was extended to the Einstein-Maxwell system by Moncrief [10]. While also starting with an expansion of the metric in spherical harmonics, Moncrief uses the ADM Hamiltonian to find a symmetric wave equation. In particular, he introduces gauge-invariant quantities and at the same time, separates the constraint variables from the dynamical variables.

As we have argued in a recent letter [11], the metric approach fails to yield hyperbolic and symmetric equations in the presence of general gravitating matter fields: In order to obtain a wave operator appearing already offshell, one has to introduce amplitudes which are adapted to the stationarity rather than the spherical symmetry of the background. Using ideas introduced by ChoquetBruhat et al. for full, non-linear general relativity [5], we show that a hyperbolic, symmetric wave equation for the extrinsic curvature can be obtained by natural means when the background is static. In contrast to the traditional, metric based approach, our curvature based approach is manifestly gauge-invariant, hyperbolic and symmetric without using the spherical symmetry of the background. More importantly, we also show that a natural generalization to self-gravitating fields is possible, including non-Abelian gauge fields and perfect fluids.

This work is organized as follows: In Section II, we show how to obtain a manifestly hyperbolic and symmetric wave operator for the perturbation of the extrinsic curvature on a static background. Then, in Section III, we specialize the result on a vacuum background and discuss the initial value formulation and the projection onto the constraint manifold. The coupling to Yang-MillsHiggs fields and to perfect fluids is discussed in Sections IV and V, respectively. In Appendix A, we show how to separate the constraint and dynamical variables and rederive the Regge-Wheeler and Zerilli equations in quite a natural way. Finally, in Appendix B, we recall a useful field theoretical formulation of perfect fluids and show how this formulation applies to perturbation theory. 


\section{THE CONSTRUCTION OF THE WAVE OPERATOR}

The goal is to linearize the equations governing selfgravitating matter fields for a static background and to bring the perturbation equations into the form of a symmetric wave equation,

$$
\left(\partial_{t}^{2}+\mathcal{A}\right) u=0
$$

where $u$ describes the perturbed gravitational and matter fields, and where $\mathcal{A}$ is a (formally) self-adjoint, elliptic operator containing spatial derivatives up to second order. In particular, this implies that equation (11) is hyperbolic, which guarantees a well-posed initial value formulation. Furthermore, as the spatial operator $\mathcal{A}$ is self-adjoint, methods form spectral theory can be applied and stability issues can be discussed analytically.

In order to give some motivation for the ideas developed below, we construct the wave operator belonging to the source-free Maxwell equations in flat space-time. In view of a generalization to non-Abelian gauge groups it is convenient to formulate Maxwell's equations in terms of the electric one-form, $E$, and the magnetic potential one-form, $A$, where $B=* d A$. Maxwell's equations constitute a system of constrained evolution equations, with Gauss constraint

$$
d^{\dagger} E=0
$$

and evolution equations

$$
\dot{A}=d \phi-E, \quad \dot{E}=d^{\dagger} d A .
$$

Here, the codifferential operator $d^{\dagger}$ for a $p$-form $\omega$ is defined by $d^{\dagger} \omega \equiv(-1)^{p} * d * \omega$. Note that there is no evolution equation for the electric scalar potential $\phi$. This reflects the gauge freedom of the theory. Usually, a wave equation for $A$ is derived by imposing the Lorentz gauge condition $\dot{\phi}+d^{\dagger} A=0$. However, $A$ is not gauge-invariant. In order to obtain a hyperbolic wave equation in terms of the gauge-invariant quantity $E$, we differentiate the second evolution equation with respect to $t$, eliminate $\dot{A}$ using the first evolution equation and add the differential of the constraint equation. This yields

$$
\ddot{E}+\left(d^{\dagger} d+d d^{\dagger}\right) E=0,
$$

which is of the desired form (1). The initial value problem may be solved as follows: First, any function $\phi(t, x)$ is chosen. Next, initial data $A_{t=0}$ and $E_{t=0}$ are given, where $E_{t=0}$ is subject to the Gauss constraint. Then, $\dot{E}_{t=0}$ is computed from the second evolution equation, where $d^{\dagger} \dot{E}_{t=0}=0$ automatically follows. In a next step, $E_{t}$ is computed for all times using the symmetric wave equation. Finally, the magnetic potential is obtained from

$$
A_{t}=A_{t=0}+\int_{0}^{t}\left(d \phi_{\tau}-E_{\tau}\right) d \tau
$$

The Gauss constraint and the second evolution equation, which was differentiated in order to get the wave operator, are both constraint equations for the initial data. It is easy to see that they propagate.

A gauge-invariant wave operator governing linear fluctuations of self-gravitating fields on a static background can be constructed in a similar manner. First, we notice that a manifestly symmetric formulation of the perturbation equations for a vacuum space-time exist:

$$
\frac{\delta\left(\sqrt{-g} G^{\mu \nu}\right)}{\sqrt{-g}}=\frac{1}{2} \eta^{\mu \alpha \sigma \tau} \eta_{\tau}^{\nu \beta \rho} \nabla_{(\alpha} \nabla_{\beta)} \delta g_{\sigma \rho}+G^{\alpha \beta \mu \nu} \delta g_{\alpha \beta} .
$$

Here, $G_{\mu \nu}$ is the Einstein tensor, $\eta$ is the volume form on $M$ and the tensor $G^{\alpha \beta \mu \nu}$ is given in terms of the Riemann tensor, $R_{\beta \mu \nu}^{\alpha}$, the Ricci tensor, $R_{\beta \nu}=R_{\beta \mu \nu}^{\mu}$, and the Ricci scalar, $R=R_{\mu}^{\mu}$, by

$$
\begin{aligned}
& G^{\alpha \beta \mu \nu}=-\frac{1}{2} R^{\alpha \mu \beta \nu} \\
& +\frac{1}{4}\left(2 g^{\alpha \beta} g^{\mu \nu}+2 g^{\mu \nu} R^{\alpha \beta}-3 g^{\alpha \mu} R^{\beta \nu}-3 g^{\beta \nu} R^{\alpha \mu}\right) \\
& +\frac{1}{4}\left(g^{\mu \alpha} g^{\nu \beta}+g^{\mu \beta} g^{\nu \alpha}-g^{\alpha \beta} g^{\mu \nu}\right) R .
\end{aligned}
$$

Clearly, the operator on the right-hand side (RHS) of Eq. (2) is formally self-adjoint with respect to the inner product

$$
\left\langle\delta g^{(1)}, \delta g^{(2)}\right\rangle=\int g^{\alpha \mu} g^{\beta \nu} \delta g_{\alpha \beta}^{(1)} \delta g_{\mu \nu}^{(2)} \eta
$$

In order to get an evolution equation for the perturbed geometry on a static background, our program is the following:

1. First, we perform a $3+1$ decomposition of Eq. (2) for a static background. The resulting equations are, of course, still symmetric with respect to the inner product (3), but split into two sets, comprising the constraint equations and the evolution equations, respectively

2. Among the 10 components of $\delta g_{\alpha \beta}, 4$ correspond to infinitesimal coordinate transformations. Hence, one has to fix this gauge, or better, to construct 6 gauge-invariant amplitudes. For a static background the components of the linearized extrinsic curvature tensor are gauge-invariant, up to a reparametrization of time. If, in addition, spacetime is spherically symmetric, full gauge-invariant quantities can be constructed from the components of the extrinsic curvature tensor.

3. The perturbation equations will be put into the desired form (11), where the spatial operator $\mathcal{A}$ is first required to be elliptic. In order to achieve this, ideas introduced by Choquet-Bruhat et. al. [5] are 
used. Extending their method, the spatial operator is finally made symmetric with respect to the inner product (3), where $g^{\alpha \mu} g^{\beta \nu}$ is replaced by its spatial part $\bar{g}^{i k} \bar{g}^{j l}$.

\section{A. The ADM equations}

The Arnowitt-Deser-Misner (ADM) formalism provides a $3+1$ decomposition of (the portion of) spacetime with topology $M \equiv \mathbb{R} \times \Sigma$, where $\Sigma$ is a threedimensional Riemannian manifold. The manifold $M$ is foliated by a one-parameter family of embeddings $e_{t}$ : $\Sigma \rightarrow M, t \in \mathbb{R}$, where the hypersurfaces $\Sigma_{t} \equiv e_{t}(\Sigma)$ are assumed to be space-like. With respect to this foliation, the timelike vector field $\partial_{t}$ is decomposed according to $\partial_{t}=\alpha n+\beta$, where $n$ is a normal unit vector field orthogonal to $\Sigma_{t}$, and $\beta$ is tangential to $\Sigma_{t}$. The metric assumes the form

$$
\boldsymbol{g}=-\alpha^{2} d t^{2}+\bar{g}_{i j}\left(d x^{i}+\beta^{i} d t\right)\left(d x^{j}+\beta^{j} d t\right)
$$

where the $x^{i}$ are local coordinates on $\Sigma$, and $\overline{\boldsymbol{g}}$ is the induced Riemannian metric on $\Sigma_{t}$. With respect to the metric (4), the Einstein tensor becomes

$$
\begin{aligned}
G_{00} & =\frac{1}{2}\left(\bar{R}-\bar{G}^{i j k l} K_{i j} K_{k l}\right), \\
G_{i 0} & =\bar{G}_{i j k l} \bar{\nabla}^{j} K^{k l},
\end{aligned}
$$

and

$$
\begin{aligned}
G_{i j} & =\bar{G}_{i j}-2 K_{i}^{s} K_{s j}+K K_{i j}+\frac{1}{2} \bar{g}_{i j}\left(3 K^{r s} K_{r s}-K^{2}\right) \\
& -\frac{1}{\alpha} \bar{G}_{i j}{ }^{k l} \bar{\nabla}_{k} \bar{\nabla}_{l} \alpha+\frac{1}{\alpha} \bar{G}_{i j}{ }^{k l}\left(\partial_{t}-\bar{L}_{\beta}\right) K_{k l}
\end{aligned}
$$

where the index zero refers to the normal vector field $e_{0}=n=\frac{1}{\alpha}\left(\partial_{t}-\beta\right)$. The $K_{i j}$ denote the components of the extrinsic curvature,

$$
K_{i j}=\frac{1}{2 \alpha}\left(\partial_{t}-\bar{L}_{\beta}\right) g_{i j}=\frac{1}{2 \alpha}\left(\partial_{t} \bar{g}_{i j}-\bar{\nabla}_{i} \beta_{j}-\bar{\nabla}_{j} \beta_{i}\right),
$$

where $K$ is the trace of $K_{i j}, K=\bar{g}^{i j} K_{i j}$. All quantities with a bar refer to the Riemannian metric $\overline{\boldsymbol{g}}$. The tensor $\bar{G}^{i j k l}$ is the De Witt metric on the space of symmetric, positive definite matrices,

$$
\bar{G}^{i j k l}=\frac{1}{2}\left(\bar{g}^{i k} \bar{g}^{j l}+\bar{g}^{i l} \bar{g}^{j k}-2 \bar{g}^{i j} \bar{g}^{k l}\right) .
$$

Equations (7) and (8) are a set of evolution equations for the 3-dimensional metric $\overline{\boldsymbol{g}}$ and the extrinsic curvature $\boldsymbol{K}$, whereas eqs. (5) and (6) are constraint equations. The freedom to choose the slicing is reflected by the fact that there are no evolution equations for the lapse, $\alpha$, and the shift, $\beta$. The Bianchi identities,

$$
0=\nabla_{\mu} G^{\mu \nu}=\partial_{0} G^{0 \nu}+\partial_{k} G^{k \nu}+\Gamma_{\mu \sigma}^{\mu} G^{\sigma \nu}+\Gamma_{\mu \sigma}^{\nu} G^{\mu \sigma}
$$

guarantee that the Hamiltonian and the momentum constraints, eqs. (5) and (6) respectively, propagate.

A convenient choice for the lapse and the shift is given by the gauge conditions

$$
K=\text { const. }, \quad \beta=0,
$$

for which the ADM equations simplify considerably. (For asymptotically flat space-times this implies the maximal slicing condition $K=0$.) In particular, one has for constant $K$

$$
\frac{\alpha}{2}\left(\bar{g}^{i j} G_{i j}-3 G_{00}\right)=\left(\bar{\Delta}-\bar{R}-K^{2}\right) \alpha
$$

In vacuum, this yields an elliptic equation for the lapse $\alpha$, which, in a numerical evolution scheme, has to be solved after each time step.

\section{B. Coordinate-invariant quantities}

In a static space-time it is convenient to choose an adapted slicing (i.e. orthogonal to $\partial_{t}$ ), such that $\beta=0$, $\partial_{t} \alpha=0$ and $\partial_{t} \bar{g}_{i j}=0$. As a consequence, the extrinsic curvature tensor vanishes,

$$
K_{i j}=0 .
$$

The metric then becomes $\boldsymbol{g}=-\alpha^{2} d t^{2}+\overline{\boldsymbol{g}}$, while the ADM equations reduce to

$$
R_{00}=\frac{1}{\alpha} \bar{\Delta} \alpha, \quad R_{0 j}=0, \quad R_{i j}=\bar{R}_{i j}-\frac{1}{\alpha} \bar{\nabla}_{i} \bar{\nabla}_{j} \alpha .
$$

Now consider a tensor field $T_{\mu \nu}$ on $M$, which is static on the background, $\dot{T}_{\mu \nu}=0, T_{t j}=T_{i t}=0$. Under an infinitesimal coordinate transformation, $x^{\mu} \mapsto x^{\mu}+\delta x^{\mu}$, generated by the vector field $X^{\mu}=\delta x^{\mu}, \delta T_{\mu \nu}$ transforms according to $\delta T_{\mu \nu} \mapsto \delta T_{\mu \nu}+L_{X} T_{\mu \nu}$. Writing $\left(X^{\mu}\right)=$ $\left(X^{t}, X^{i}\right)=\left(f, X^{i}\right)$, one has

$$
\begin{aligned}
& \delta T_{t t} \mapsto \delta T_{t t}+X^{i} T_{t t, i}+2 \dot{f} T_{t t}, \\
& \delta T_{t j} \mapsto \delta T_{t j}+\dot{X}^{i} T_{i j}+f_{j} T_{t t}, \\
& \delta T_{i j} \mapsto \delta T_{i j}+X^{s} \bar{\nabla}_{s} T_{i j}+T_{i s} \bar{\nabla}_{j} X^{s}+T_{s j} \bar{\nabla}_{i} X^{s},
\end{aligned}
$$

where here and in the following a dot denotes differentiation with respect to $t$, and $f_{j} \equiv \partial_{j} f$. Using $\delta g_{t t}=-2 \alpha \delta \alpha$ and $\delta g_{t j}=\delta \beta_{j}$, as well as the formulae $\alpha^{2} \delta T_{00}=\delta T_{t t}-2 T_{t t} \delta \alpha / \alpha, \alpha \delta T_{0 j}=\delta T_{t j}-T_{i j} \delta \beta^{i}$, the metric and the Einstein tensor transform according to

$$
\begin{aligned}
& \delta \alpha \mapsto \delta \alpha+\alpha_{i} X^{i}+\alpha \dot{f}, \\
& \delta \beta_{j} \mapsto \delta \beta_{j}+\dot{X}_{j}-\alpha^{2} f_{j}, \\
& \delta \bar{g}_{i j} \mapsto \delta \bar{g}_{i j}+\bar{\nabla}_{i} X_{j}+\bar{\nabla}_{j} X_{i},
\end{aligned}
$$


and

$$
\begin{aligned}
\delta G_{00} & \mapsto \delta G_{00}+G_{00, i} X^{i}, \\
\delta G_{0 j} & \mapsto \delta G_{0 j}+\alpha\left(G_{00} f_{j}+G_{i j} f^{i}\right), \\
\delta G_{i j} & \mapsto \delta G_{i j}+X^{s} \bar{\nabla}_{s} G_{i j}+G_{i s} \bar{\nabla}_{j} X^{s}+G_{s j} \bar{\nabla}_{i} X^{s} .
\end{aligned}
$$

The above transformation properties imply that one can construct "vector-invariant" quantities, i.e., quantities which are invariant under the subset of transformations generated by vector fields $\left(X^{\mu}\right)=\left(f, X^{i}\right)$ with $f=0$ :

$$
\begin{aligned}
\delta \dot{G}_{00}^{(i n v)} & \equiv \delta \dot{G}_{00}-G_{00, i} \delta \beta^{i} \\
\delta G_{0 j}^{(i n v)} & \equiv \delta G_{0 j} \\
\delta \dot{G}_{i j}^{(i n v)} & \equiv \delta \dot{G}_{i j}-\delta \beta^{s} \bar{\nabla}_{s} G_{i j}-G_{s i} \bar{\nabla}_{j} \delta \beta^{s}-G_{s j} \bar{\nabla}_{i} \delta \beta^{s},
\end{aligned}
$$

and

$$
\begin{aligned}
\delta \dot{\alpha}^{(i n v)} & \equiv \delta \dot{\alpha}-\alpha^{j} \delta \beta_{j}, \\
\delta \dot{\bar{g}}_{i j}^{(i n v)} & \equiv \delta \dot{\bar{g}}_{i j}-\bar{\nabla}_{i} \delta \beta_{j}-\bar{\nabla}_{j} \delta \beta_{i} .
\end{aligned}
$$

The fact that $\delta \dot{\bar{g}}_{i j}^{(i n v)}=2 \alpha \delta K_{i j}$ suggests that - from the ADM point of view - the natural gauge-invariant perturbations on a static background are the extrinsic curvatures, rather than the metric fields. We are, therefore, looking for a symmetric wave equation in terms of the perturbed extrinsic curvature tensor $\delta K_{i j}$.

\section{The linearized ADM equation}

The derivation of the linearized field equations is considerably simplified by choosing an appropriate gauge. For a static background the vector field $X^{j}$ in Eq. (13) can be arranged such that $\delta \beta^{j}=0$. (Note, however, that this gauge is not adapted to describe stationary perturbations.) In this gauge, the perturbed quantities $\delta \dot{\alpha}, \delta \dot{\bar{g}}$ and $\delta \dot{G}_{\mu \nu}$ coincide with their vector-invariant counterparts constructed above. The remaining residual gauge freedom is generated by the vector field $\left(X^{\mu}\right)=\left(f, X^{i}\right)$ with $\dot{X}_{j}=\alpha^{2} f_{j}$,

$$
\begin{gathered}
\delta \dot{\alpha} \mapsto \delta \dot{\alpha}+\alpha^{2} \alpha_{j} f^{j}+\alpha \ddot{f}, \\
\delta K_{i j} \mapsto \delta K_{i j}+\frac{1}{\alpha} \bar{\nabla}_{(i}\left(\alpha^{2} \bar{\nabla}_{j)} f\right) .
\end{gathered}
$$

Here and in the following, we use the notation $2 \omega_{(i j)} \equiv$ $\omega_{i j}+\omega_{j i}$.

Using the fact that quadratic terms in $\boldsymbol{K}$ give no contributions if the background is static, the linearized ADM constraint equations become

$$
\delta G_{00}=\frac{1}{2} \delta \bar{R}, \quad \delta G_{i 0}=\bar{G}_{i j k l} \bar{\nabla}^{j} \delta K^{k l},
$$

while the evolution equations are

$$
\begin{aligned}
\delta K_{i j} & =\frac{1}{2 \alpha} \delta \dot{\bar{g}}_{i j} \\
\delta G_{i j} & =\delta \bar{G}_{i j}-\delta \frac{1}{\alpha} \bar{G}_{i j}{ }^{k l} \bar{\nabla}_{k} \bar{\nabla}_{l} \alpha+\frac{1}{\alpha} \bar{G}_{i j}{ }^{k l} \delta \dot{K}_{k l} .
\end{aligned}
$$

A wave equation for $\delta \boldsymbol{K}$ is obtained as follows: One first differentiates Eq. (19) with respect to $t$ and uses Eq. (18) to eliminate $\delta \dot{\bar{g}}$. Applying the general formulae

$$
\begin{aligned}
2 \delta R_{\alpha \beta} & =-\nabla^{\mu} \nabla_{\mu} \delta g_{\alpha \beta}-\nabla_{\alpha} \nabla_{\beta}\left(g^{\mu \nu} \delta g_{\mu \nu}\right) \\
& +\nabla^{\mu} \nabla_{\beta} \delta g_{\alpha \mu}+\nabla^{\mu} \nabla_{\alpha} \delta g_{\beta \mu}
\end{aligned}
$$

and

$$
\delta G_{\alpha \beta}=\delta R_{\alpha \beta}-\frac{1}{2} R \delta g_{\alpha \beta}-\frac{1}{2} g_{\alpha \beta}\left(g^{\mu \nu} \delta R_{\mu \nu}-R^{\mu \nu} \delta g_{\mu \nu}\right)
$$

to the variation of the Einstein tensor $\bar{G}_{i j}$ in three dimensions, and also using the background equations (11), then yields the following expressions in terms of the vectorinvariant amplitudes:

$$
L_{i j} \equiv \alpha \delta K_{i j}=\frac{1}{2} \delta \dot{g}_{i j}, \quad A \equiv \delta \dot{\alpha},
$$

$$
\begin{aligned}
S_{i j} & \equiv \frac{\alpha}{\sqrt{-g}} \partial_{t} \delta\left(\sqrt{-g} G_{i j}\right) \\
& =S_{i j}^{B G}+\bar{G}_{i j}{ }^{k l} \square L_{k l}+2 \alpha \bar{R}^{k}{ }_{i} L_{j) k}-2 \alpha \bar{R}_{k i l j} L^{k l} \\
& +2 \bar{\nabla}_{(i} \alpha \bar{\nabla}^{k} L_{j) k}-2 \bar{\nabla}^{k}\left(\alpha_{(i} L_{j) k}\right)+2 \alpha^{k} \bar{\nabla}_{(i} L_{j) k} \\
& +2\left(\bar{\nabla}_{(i} \bar{\nabla}^{k} \alpha\right) L_{j) k}-\alpha \bar{\nabla}_{i} \bar{\nabla}_{j} L-\bar{g}_{i j} \bar{\nabla}^{k} \bar{\nabla}^{l}\left(\alpha L_{k l}\right) \\
& -\frac{1}{\alpha} \bar{G}_{i j}{ }^{k l} \bar{\nabla}_{(k} \alpha^{2} \bar{\nabla}_{l)}\left(\frac{A}{\alpha}\right),
\end{aligned}
$$

where the d'Alembertian is defined according to

$$
\square=\frac{1}{\alpha} \partial_{t}^{2}-\bar{\nabla}^{r} \alpha \bar{\nabla}_{r},
$$

and where

$$
\begin{aligned}
S_{i j}^{B G} & \equiv \alpha G_{i j} L+\bar{g}_{i j} \alpha G^{k l} L_{k l}-\alpha R L_{i j}+\frac{1}{2} \bar{g}_{i j} \alpha R L \\
& +G_{i j} A .
\end{aligned}
$$

In addition, we consider the equation

$$
\begin{aligned}
S_{00} & \equiv \frac{-1}{\sqrt{-g}} \partial_{t} \delta\left(\sqrt{-g} G_{00}\right) \\
& =-\frac{1}{\alpha} G_{00} A-\frac{1}{\alpha} \bar{G}^{i j k l} \bar{\nabla}_{i} \alpha^{2} \bar{\nabla}_{j}\left(\frac{L_{k l}}{\alpha}\right)+G^{i j} L_{i j} .
\end{aligned}
$$

As expected from the general formula (2), the spatial part of the operators in $\left(S_{00}, S_{i j}\right)$ is symmetric in $u=\left(A, L_{i j}\right)$ with respect to the inner product

$$
\left\langle u^{(1)}, u^{(2)}\right\rangle=\int_{\Sigma}\left(A^{(1)} A^{(2)}+\bar{g}^{i k} \bar{g}^{j l} L_{i j}^{(1)} L_{k l}^{(2)}\right) \bar{\eta} .
$$


Furthermore, one has the following constraint equation for $L_{i j}$ :

$$
\delta G_{i 0}=\bar{G}_{i j k l} \bar{\nabla}^{j}\left(\frac{L^{k l}}{\alpha}\right) .
$$

The system $S_{i j}$ still has the following drawbacks: First, the spatial operator is not elliptic: Considering, in particular, perturbations of the form $L_{k l}=\frac{1}{3} L \bar{g}_{i j}$, the second derivatives yield $-\frac{\alpha}{3}\left(\bar{\nabla}_{i} \bar{\nabla}_{j}-\bar{g}_{i j} \bar{\Delta}\right) L$, which is not governed by an elliptic operator. Second, the "kinematic energy metric", $\bar{G}^{i j k l}$, is not positive. Indeed, for a symmetric tensor field $t_{i j}=\hat{t}_{i j}+\frac{1}{3} \bar{g}_{i j} t$, where $\hat{t}_{i j}$ is trace-less, one finds $\bar{G}_{i j}{ }^{k l} t_{k l}=\hat{t}_{i j}-\frac{2}{3} \bar{g}_{i j} t$. Finally, there exists no evolution equation for the perturbed lapse $A$.

In order to overcome these problems, we first apply ideas introduced in [5], where it was shown that the spatial operator becomes elliptic after using the momentum constraint (25) and its spatial derivatives. Slightly changing the procedure outlined in [5], this can be achieved without destroying the symmetry of the final operator. It turns out that the combination

$$
\begin{aligned}
\Lambda_{i j} & \equiv \frac{\alpha}{\sqrt{-g}} \partial_{t} \delta\left(\sqrt{-g} G_{i j}\right)-\frac{2}{\alpha} \bar{\nabla}_{(i}\left(\alpha^{3} \delta G_{j) 0}\right) \\
& +\frac{1}{\alpha^{2}} \bar{g}_{i j} \bar{\nabla}^{k}\left(\alpha^{4} \delta G_{k 0}\right)
\end{aligned}
$$

almost yields the desired result: $\Lambda_{i j}=\Lambda_{i j}^{V}+S_{i j}^{B G}$, where

$$
\begin{aligned}
\Lambda_{i j}^{V} & =\bar{G}_{i j}{ }^{k l} \square L_{k l}+2 \alpha \bar{R}_{(i}^{k} L_{j) k}-2 \alpha \bar{R}_{k i l j} L^{k l} \\
& +4 \bar{\nabla}_{(i}\left(\alpha^{k} L_{j) k}\right)-4 \alpha_{(i} \bar{\nabla}^{k} L_{j) k}-2 \alpha \bar{\nabla}^{k}\left(\frac{\alpha_{(i}}{\alpha}\right) L_{j) k} \\
& -\frac{2}{\alpha} \bar{\nabla}_{i}\left(\alpha \alpha_{j}\right) L-\frac{2}{\alpha} \bar{g}_{i j} \bar{\nabla}^{k}\left(\alpha \alpha^{l}\right) L_{k l}-2 \alpha \bar{g}_{i j} \bar{\nabla}^{k}\left(\frac{\alpha_{k}}{\alpha}\right) L \\
& +\frac{1}{\alpha} \bar{G}_{i j}{ }^{k l}\left[\bar{\nabla}_{(k} \alpha^{2} \bar{\nabla}_{l)}\left(L-\frac{1}{\alpha} A\right)\right] .
\end{aligned}
$$

(The main difference between $\Lambda_{i j}$ and the $\Omega_{i j}$ introduced in [5] lies in the symmetrizing terms.)

In order to obtain a symmetric wave equation, one makes use of the residual gauge freedom (16) and adopts one of the following two gauges: The first possibility is to choose the harmonic gauge

$$
A=\alpha L,
$$

in which case $\Lambda_{i j}^{V}$ becomes a symmetric operator in $L_{i j}$. Furthermore, this also solves the lapse-problem. Unfortunately, the De Witt metric still appears in front of the d'Alembertian $\square$, and, as a consequence, the spatial operator is not elliptic. Alternatively, one can multiply $\Lambda_{i j}^{V}$ from the left by the inverse of the De Witt metric,

$$
\bar{G}_{i j k l}^{-1}=\frac{1}{2}\left(\bar{g}_{i k} \bar{g}_{j l}+\bar{g}_{i l} \bar{g}_{j k}-\bar{g}_{i j} \bar{g}_{k l}\right),
$$

which yields a hyperbolic wave-operator, which is, however, only symmetric with respect to the inner product induced by the indefinite de Witt metric $\bar{G}^{i j k l}$. This possibility has been considered in 11].

In order to get a positive kinematic energy metric, it seems unavoidable to choose the maximal (or constant mean curvature) slicing condition,

$$
L=\bar{g}^{i j} L_{i j}=0 .
$$

(This gauge may not always exist if the background manifold $\Sigma$ is closed. In fact, Eq. (16) shows that one has to solve an elliptic equation for $f$ in order to get $L=0$.) Adopting the maximal gauge, the trace-less part of $\Lambda_{i j}$ yields a manifestly hyperbolic, self-adjoint operator for the symmetric, trace-less tensor $L_{i j}$. However, the perturbations of the lapse are still present in this gauge. The amplitude $A$ obeys an elliptic equation which corresponds to the trace of $\Lambda_{i j}^{V}$. Hence, the system is of mixed hyperbolic-elliptic type. However, as will be shown below, all terms involving $A$ disappear when the operator in $\Lambda_{i j}$ is protected onto the constraint manifold defined by (25).

\section{The linearized Bianchi identities}

In the following the linearized version of the Bianchi identities (9) will be needed. In particular, we want to show explicitely that the constraint equation (25) propagates. The ADM metric (4) with $\beta=0$ has the following Christoffel symbols:

$$
\begin{aligned}
& \Gamma_{t t}^{t}=\frac{\dot{\alpha}}{\alpha}, \quad \Gamma_{t j}^{t}=\frac{\alpha_{j}}{\alpha}, \quad \Gamma_{i j}^{t}=\frac{1}{\alpha} K_{i j}, \\
& \Gamma_{t t}^{k}=\alpha \alpha^{k}, \quad \Gamma_{t j}^{k}=\alpha K_{j}^{k}, \quad \Gamma_{i j}^{k}=\bar{\Gamma}_{i j}^{k} .
\end{aligned}
$$

Using this in Eq. (9) yields the $3+1$ decomposition of the Bianchi identities:

$$
\begin{aligned}
0 & =\nabla^{\mu} G_{\mu t} \\
& =-\dot{G}_{00}-\alpha K G_{00}+\frac{1}{\alpha} \bar{\nabla}^{k}\left(\alpha^{2} G_{0 k}\right)-\alpha G^{k l} K_{k l}, \\
0 & =\alpha \nabla^{\mu} G_{\mu j} \\
& =-\dot{G}_{0 j}+G_{00} \alpha_{j}-G_{0 j} \alpha K+\bar{\nabla}^{k}\left(\alpha G_{k j}\right) .
\end{aligned}
$$

Linearizing Eq. (29) on a static background yields

$$
\delta \dot{G}_{00}-\frac{1}{\alpha} \bar{\nabla}^{k}\left(\alpha^{2} \delta G_{0 k}\right)=-\alpha G^{k l} \delta K_{k l}-\alpha G_{00} \delta K,
$$

which shows that no new constraint equations for $\delta K_{i j}$ are obtained when the Hamilton constraint is differentiated with respect to $t$.

Using Eq. (31), the linearized version of Eq. (30) becomes

$$
\begin{aligned}
0 & =\square C_{j}-\alpha \bar{R}_{j k} C^{k}+2 \bar{\nabla}_{j}\left(\alpha^{k} C_{k}\right)-2 \alpha_{j} \bar{\nabla}^{k} C_{k} \\
& -\alpha \bar{\nabla}^{k}\left(\frac{\alpha_{k}}{\alpha}\right) C_{j}-\frac{1}{\alpha} \bar{\nabla}^{k}\left(\alpha \alpha_{j}\right) C_{k}-\alpha \bar{\nabla}^{k} \Lambda_{k j}+(B G),
\end{aligned}
$$


where $\Lambda_{i j}$ is given in (26). The constraint variables $C_{j}$ are defined according to

$$
C_{j} \equiv \alpha^{2} \delta G_{0 j},
$$

and $(B G)$ denotes terms which are proportional to components of the Einstein tensor. The fact that Eq. (32) also holds for the stress-energy tensor if the matter equations are satisfied, implies that these terms need not be considered any longer, provided that $\delta G_{\mu \nu}$ is replaced by $\delta G_{\mu \nu}-8 \pi G \delta T_{\mu \nu}$ in Eqs. (32) and the definition of the $C_{j}$.

Hence, if the evolution equations, $\Lambda_{i j}=0$, and the background equations are fulfilled, it follows that the constraints $C_{j}$ propagate. Note that the operator governing the $C_{j}$ in Eq. (32) is hyperbolic and symmetric.

\section{E. Summary}

The linearized Einstein equations for a static background yield a hyperbolic, formally self-adjoint operator for the variation of the extrinsic curvature. This quantity is coordinate-invariant with respect to infinitesimal diffeomorphisms within the slices orthogonal to $\partial_{t}$. The wave operator is constructed from the combination $\Lambda_{i j}$ defined in (26). In the maximal slicing gauge, $\delta K=0$, this yields (using also the background equation $\left.R_{00}=\frac{1}{\alpha} \bar{\Delta} \alpha\right)$

$$
\Lambda_{k}^{k}=\bar{g}^{k l} S_{k l}^{(B G)}+2\left(\bar{\Delta}-R_{00}\right) A-\frac{4}{\alpha} \bar{\nabla}^{k}\left(\alpha \alpha^{l}\right) L_{k l},
$$

and

$$
\begin{aligned}
\hat{\Lambda}_{i j} & =\hat{S}_{i j}^{(B G)}+\square L_{i j}+2 \alpha \bar{R}_{(i}^{k} L_{j) k}-2 \alpha \bar{R}_{k i l j} L^{k l} \\
& +4 \bar{\nabla}_{(i}\left(\alpha^{k} L_{j) k}\right)-4 \alpha_{(i} \bar{\nabla}^{k} L_{j) k}+2 \alpha \bar{\nabla}^{k}\left(\frac{\alpha_{(i}}{\alpha}\right) L_{j) k} \\
& -\frac{1}{\alpha} \bar{\nabla}_{(i} \alpha^{2} \bar{\nabla}_{j)}\left(\frac{A}{\alpha}\right) \\
& +\frac{1}{3} \bar{g}_{i j}\left(-\frac{2}{\alpha} \bar{\nabla}^{k}\left(\alpha \alpha^{l}\right) L_{k l}+\bar{\Delta} A-R_{00} A\right)
\end{aligned}
$$

where $\hat{\Lambda}_{i j}$ denotes the trace-less part of $\Lambda_{i j}$, and where the trace-less symmetric tensor $L_{i j}$ and the scalar $A$ are defined by

$$
L_{i j} \equiv \alpha \delta K_{i j}, \quad A \equiv \delta \dot{\alpha} .
$$

These quantities are subject to the residual gauge transformation (16) with $\bar{\nabla}^{k}\left(\alpha^{2} \bar{\nabla}_{k} f\right)=0$. The system $\left(\hat{\Lambda}_{i j}, \Lambda_{k}^{k}\right)$ provides a closed set of evolution equations for $\left(L_{i j}, A\right)$, which is manifestly hyperbolic and symmetric in $L_{i j}$ and elliptic in $A$. The operator $-\bar{\Delta}+R_{00}$ in equation (33) is non-negative if the strong energy condition and the background equations hold, i.e., if $R_{00} \geq 0$.

Furthermore, $L_{i j}$ obeys the momentum constraint equation

$$
\delta G_{i 0}=\bar{\nabla}^{j}\left(\frac{L_{i j}}{\alpha}\right) .
$$

The constraint variables,

$$
C_{j}=\alpha^{2}\left(\delta G_{0 j}-8 \pi G T_{0 j}\right),
$$

propagate by virtue of Eq. (32).

Finally, the differentiated Hamilton constraint yields

$$
\begin{aligned}
\Lambda_{00} & \equiv \frac{-1}{\sqrt{-g}} \partial_{t} \delta\left(\sqrt{-g} G_{00}\right) \\
& =-\frac{1}{\alpha} G_{00} A-\frac{1}{\alpha} \bar{\nabla}^{i} \alpha^{2} \bar{\nabla}^{j}\left(\frac{L_{i j}}{\alpha}\right)+G^{i j} L_{i j},
\end{aligned}
$$

and the system $\left(\Lambda_{00}, \hat{\Lambda}_{i j}\right)$ is symmetric in $\left(A, L_{i j}\right)$ with respect to the inner product defined in (24).

\section{THE VACUUM EQUATIONS}

We start by analyzing the wave operator for vacuum perturbations of a static vacuum space-time. By virtue of the constraint equation

$$
0=\bar{\nabla}^{j}\left(\frac{L_{i j}}{\alpha}\right),
$$

the equation $\Lambda_{00}=0$ is fulfilled and Eq. (33) can be rewritten as

$$
\bar{\Delta} A=2 \bar{\nabla}^{k}\left(\alpha^{l} L_{k l}\right) .
$$

The Laplacian on the left-hand side (LHS) is elliptic and symmetric on the dense subspace of $C^{\infty}$-functions in the Hilbert space $L^{2}(\Sigma, \bar{\eta})$. As a consequence, its image is equal to the orthogonal complement of its kernel. If $\Sigma$ is compact, the kernel ker $\bar{\Delta}$ is the set of constant functions on $\Sigma$. If $\Sigma$ is not compact, but all perturbations vanish sufficiently fast at space-like infinity, $\operatorname{ker} \bar{\Delta}$ is trivial. In either case, the RHS of (37) is orthogonal to ker $\bar{\Delta}$ and therefore lies in the image of $\bar{\Delta}$. This shows that equation (37) is solvable. A solution, which is unique up to the addition of an element in $\operatorname{ker} \bar{\Delta}$, can be written formally as

$$
A=2 \underline{\bar{\Delta}}^{-1} \bar{\nabla}^{k}\left(\alpha^{l} L_{k l}\right),
$$

where $\bar{\Delta}$ denotes the restriction of $\bar{\Delta}$ on the orthogonal complement of ker $\bar{\Delta}$.

\section{A. The initial value formulation}

A necessary condition for Einstein's equations to hold is that the evolution equations $\hat{\Lambda}_{i j}=0$ and the constraint equations (36) are satisfied. In order to find a solution of Einstein's equations, these equations must, however, be supplemented with 


$$
\begin{aligned}
& 0=\delta G_{00}=\frac{1}{2} \delta \bar{R}=\frac{1}{2 \alpha} \bar{G}^{i j k l} \bar{\nabla}_{i} \alpha^{2} \bar{\nabla}_{j}\left(\frac{\delta \bar{g}_{k l}}{\alpha}\right), \\
& 0=\delta G_{i j}=\delta \bar{G}_{i j}-\delta \frac{1}{\alpha} \bar{G}_{i j}{ }^{k l} \bar{\nabla}_{k} \bar{\nabla}_{l} \alpha+\frac{1}{\alpha^{2}} \dot{L}_{i j} .
\end{aligned}
$$

The first equation is the linearized Hamilton constraint, while the second equation is the evolution equation which earlier was differentiated with respect to $t$ to construct the wave operator.

The linearized Einstein equations are solved as follows:

1. Specify any 3 -metric $\delta \bar{g}_{i j}=\delta \bar{g}(t=0)_{i j}$ and any symmetric, trace-less tensor field $L_{i j}$ on an initial time-slice $\Sigma_{0}$, such that the Hamilton-constraint (39) and the momentum constraint (36) hold.

2. Choose any convenient function $\delta \alpha=\delta \alpha(t=0)$ on $\Sigma_{0}$.

3. Compute $A$ and $\dot{L}_{i j}$ on $\Sigma_{0}$ from equation (38) and (40), respectively. By virtue of the background equations and the linearization of the Bianchi identity $30, \dot{L}_{i j} / \alpha$ is trace- and divergence-free. Therefore, the momentum constraint propagates as a consequence of Eq. (32).

4. Evolve $\left(A, L_{i j}\right)$ via the equation $\hat{\Lambda}_{i j}=0$ and equation $(38)$.

5. The linearized metric coefficients are eventually obtained from

$$
\begin{aligned}
& \delta \alpha(t)=\delta \alpha(0)+\int_{0}^{t} A(\tau) d \tau, \\
& \delta \bar{g}(t)_{i j}=\delta \bar{g}(0)_{i j}+\int_{0}^{t} 2 L(\tau)_{i j} d \tau .
\end{aligned}
$$

Note that in contrast to the full non-linear constraint equations, the linearized Hamilton and momentum constraint decouple. (This follows from the fact that $\boldsymbol{K}$ vanishes on the background.) The "linearized" conformal method to solve the Hamilton constraint is to split

$$
\delta \bar{g}_{i j}=\hat{m}_{i j}+\frac{1}{3} \bar{g}_{i j} \phi
$$

in its trace and trace-less parts. Inserting this into Eq. (39) yields

$$
\frac{1}{\alpha} \bar{\nabla}^{k} \alpha^{2} \bar{\nabla}_{k}\left(\frac{\phi}{\alpha}\right)=\frac{3}{2} \frac{1}{\alpha} \bar{\nabla}^{k} \alpha^{2} \bar{\nabla}^{l}\left(\frac{\hat{m}_{k l}}{\alpha}\right) .
$$

Again, the RHS of this equation is orthogonal to the kernel of the operator on the LHS, which is symmetric and elliptic. Therefore, the trace-less part $\hat{m}_{i j}$ can be specified freely, and the above equation can be solved for $\phi$. How to solve the linearized momentum constraint is explained below.

\section{B. The projection onto the constraint manifold}

Our next aim is to extract the "pure dynamical degrees of freedom" by solving the momentum constraint, and to project the wave operator onto the space of dynamical variables. For vacuum perturbations this can be achieved by using the "York decomposition" [12].

First, we replace $L_{i j}$ by

$$
Z_{i j}=\frac{1}{\sqrt{\alpha}} L_{i j}
$$

and the operator $\hat{\Lambda}_{i j}$ by $\sqrt{\alpha} \hat{\Lambda}_{i j}$. This eliminates the factor $1 / \alpha$ in front of the second time derivatives in $\hat{\Lambda}_{i j}$. The linearized momentum constraint now reads

$$
\bar{\nabla}^{j}\left(\frac{Z_{i j}}{\sqrt{\alpha}}\right)=0 .
$$

Let $\mathcal{T}$ denote the space of all $C^{\infty}$ symmetric, trace-less covariant tensor fields on $\Sigma$, and let $\mathcal{V}$ denote the space of all $C^{\infty}$ vector fields on $\Sigma$. We assume that either $\Sigma$ is compact or all tensor fields vanish sufficiently rapid on the "border" of $\Sigma$. Consider the operator

$$
\begin{aligned}
& \boldsymbol{L}^{\dagger}: \mathcal{T} \longrightarrow \mathcal{V}, \\
& \left(\boldsymbol{L}^{\dagger} Z\right)_{i}=2 \bar{\nabla}^{j}\left(\frac{Z_{i j}}{\sqrt{\alpha}}\right),
\end{aligned}
$$

and let $W \in \mathcal{V}$ and $Z \in \mathcal{T}$. With respect to the inner products on $\mathcal{V}$ and $\mathcal{T}$ induced by $\bar{g}_{i j}$, we have

$$
\left\langle W, \boldsymbol{L}^{\dagger} Z\right\rangle=\langle\boldsymbol{L} W, Z\rangle,
$$

where $\boldsymbol{L}: \mathcal{V} \longrightarrow \mathcal{T}$ is given by

$$
(\boldsymbol{L} W)_{i j}=-\frac{1}{\sqrt{\alpha}}\left(\bar{\nabla}_{i} W_{j}+\bar{\nabla}_{j} W_{i}-\frac{2}{3} \bar{g}_{i j} \bar{\nabla}^{k} W_{k}\right) .
$$

The operator $\boldsymbol{L}^{\dagger} \boldsymbol{L}: \mathcal{V} \longrightarrow \mathcal{V}$ is symmetric, positive semidefinite and elliptic. Its kernel $\operatorname{ker}\left(\boldsymbol{L}^{\dagger} \boldsymbol{L}\right)=\operatorname{ker} \boldsymbol{L}$ consists of all conformal Killing vector fields on $\Sigma$. As a consequence, we can solve the equation

$$
\boldsymbol{L}^{\dagger} \boldsymbol{L} W=\boldsymbol{L}^{\dagger} Z,
$$

for given $Z \in \mathcal{T}$,

$$
W={\underline{\left(\boldsymbol{L}^{\dagger} \boldsymbol{L}\right)^{-1}}}^{\dagger} Z+W_{0},
$$

where $\left(\boldsymbol{L}^{\dagger} \boldsymbol{L}\right)$ denotes the restriction of $\boldsymbol{L}^{\dagger} \boldsymbol{L}$ on the orthogonal complement of its kernel, and where $W_{0} \in$ ker $\boldsymbol{L}$.

The orthogonal projector on the constraint manifold

$$
\mathcal{C}=\left\{\left(Z_{i j}\right) \in \mathcal{T} \mid \bar{\nabla}^{j}\left(\frac{Z_{i j}}{\sqrt{\alpha}}\right)=0\right\}=\operatorname{ker} \boldsymbol{L}^{\dagger}
$$

can therefore be represented as 


$$
\boldsymbol{P}=\mathbb{1}-\underline{\boldsymbol{L}}{\underline{\left(\boldsymbol{L}^{\dagger} \boldsymbol{L}\right)}}^{-1} \boldsymbol{L}^{\dagger}
$$

Any symmetric, trace-less tensor field $Z \in \mathcal{T}$ can be decomposed according to

$$
Z_{i j}=Z_{i j}^{T T}+Z_{i j}^{T L},
$$

where $Z^{T T} / \sqrt{\alpha}=\boldsymbol{P} Z / \sqrt{\alpha}$ is trace-less and divergencefree, and where

$$
Z_{i j}^{T L}=\underline{\boldsymbol{L}}^{\left(\boldsymbol{L}^{\dagger} \boldsymbol{L}\right)}{ }^{-1} \boldsymbol{L}^{\dagger} Z_{i j} \equiv(\boldsymbol{L} W)_{i j}
$$

is the trace-less longitudinal part. This decomposition is covariant and orthogonal with respect to the metric on $\mathcal{T}$ induced by $\bar{g}_{i j}$. For $\alpha=1$, it corresponds to York's decomposition for symmetric tensor fields $K_{i j}$. York's decomposition has further conformal properties which play an essential role in solving the full non-linear constraint equations [12].

In our case, the linearized momentum constraint is solved by taking

$$
Z_{i j}=\boldsymbol{P} \tilde{Z}_{i j}
$$

where $\tilde{Z}_{i j}$ is any symmetric, trace-less tensor field on $\Sigma$. Of course, this requires the computation of $\left(\boldsymbol{L}^{\dagger} \boldsymbol{L}\right)^{-1}$, which is a non-local operator.

Consider now the wave operator $\sqrt{\alpha} \hat{\Lambda}_{i j}$ defined in (34). It has the form

$$
\sqrt{\alpha} \hat{\Lambda}_{i j}=\left(\partial_{t}^{2}+\mathcal{A}\right) Z_{i j}+b_{i j}(A),
$$

where $\mathcal{A}$ is a spatial, elliptic and self-adjoint operator and the functions $b_{i j}(A)$ are given by

$$
b_{i j}(A)=-\frac{1}{\sqrt{\alpha}} \bar{\nabla}_{(i} \alpha^{2} \bar{\nabla}_{j)}\left(\frac{A}{\alpha}\right)+\frac{\sqrt{\alpha}}{3} \bar{g}_{i j} \bar{\Delta} A .
$$

By virtue of the linearized Bianchi identity (32), it follows that the spatial operator defined by

$$
Z_{i j} \mapsto \mathcal{A} Z_{i j}+b_{i j}(A),
$$

where $A$ is given by (38), maps the constraint manifold $\mathcal{C}$ into itself. (In particular, this implies that the momentum constraint propagates.) As a consequence, we have

$$
\mathcal{A} \boldsymbol{P} Z_{i j}+b_{i j}(A)=\boldsymbol{P}\left(\mathcal{A} \boldsymbol{P} Z_{i j}+b_{i j}(A)\right)
$$

(still provided that equation (38) holds). Now it easy to see that the $b_{i j}$ map into a space which is orthogonal to the constraint manifold. Indeed, the spatial operator in the system $\sqrt{\alpha}\left(\Lambda_{00}, \hat{\Lambda}_{i j}\right)$ can be shown to have the form

$$
\left(\begin{array}{ll}
0 & b^{\dagger} \\
b & \mathcal{A}
\end{array}\right)
$$

As mentioned above, $b^{\dagger} \boldsymbol{P}=0$, also implying that $\boldsymbol{P} b=$ 0 . Thus, the wave equation restricted to the constraint manifold takes the form of a symmetric wave equation,

$$
\left(\boldsymbol{P} \partial_{t}^{2}+\tilde{\mathcal{A}}\right) Z_{i j}=0
$$

where $\tilde{\mathcal{A}} \equiv \boldsymbol{P} \mathcal{A} \boldsymbol{P}$ is a (formally) self-adjoint spatial operator.

In conclusion, we have shown that the wave operator, projected onto the (momentum) constraint manifold, yields a symmetric wave equation for the symmetric, trace-less tensor $Z_{i j}=\sqrt{\alpha} \delta K_{i j}$. As expected, the variation of the lapse does not appear in this equation. The following two difficulties remain: First, the quantity $Z_{i j}$ is not coordinate-invariant with respect to reparametrization of time. Hence, we have not yet isolated all physical degrees of freedom. The second difficulty is to find an explicit characterization of the constraint manifold in order to have an explicit representation of the operator $\tilde{\mathcal{A}}$. We have not yet solved these problems for an arbitrary static background. However, the additional structure provided by a spherically symmetric background, enables one to solve both problems. This is discussed in Appendix A, where a natural derivation of the Regge-Wheeler and Zerilli equations is given.

\section{Locally flat space-times}

To conclude this section, we briefly specialize to the case where $\alpha \equiv 1$. Using the background equations (11), this implies that $(\Sigma, \bar{g})$ is locally flat. Equation (33) then yields $A=A(t)$, and the pulsation equation reduces to

$$
0=\hat{\Lambda}_{i j}=\left(\partial_{t}^{2}-\bar{\Delta}\right) L_{i j},
$$

with the constraint

$$
0=\bar{\nabla}^{j} L_{i j} .
$$

These equations are well-known from the weak-field limit of general relativity. They can be solved by Fourier transformation. In particular, it follows that all vacuum spacetimes, which can be represented by $I \times \Sigma$ with $I \subseteq \mathbb{R}$ and $\Sigma$ locally flat, are linearly stable.

\section{THE COUPLING TO YANG-MILLS-HIGGS FIELDS}

An important new feature of the formulation presented in this paper is the fact that it extends to gravitating matter fields in a natural way. As mentioned earlier, this is due to that - in contrast to the traditional metric approach - the wave operator governing the variation of the extrinsic curvature appears already off-shell.

In this section, we show that the equations governing fluctuations of a static solution to the EinsteinYang-Mills-Higgs (EYMH) equations assume the form of a symmetric wave equation. While this is explicitely established for the triplet case, we emphasize that it holds true whenever the gauge group is a compact Lie group. (Compactness guarantees the existence of an Adinvariant scalar product on the Lie algebra.) 


\section{A. The ADM equations}

In the $\mathrm{ADM}$ formalism, the gauge potential $A$ is parametrized in terms of a scalar field $\Phi \equiv-i_{\partial_{n}} A$ and a one-form $\bar{A}$ (both Lie algebra valued),

$$
A=-\Phi \alpha d t+\bar{A}_{i}\left(d x^{i}+\beta^{i} d t\right) .
$$

Similarly to the extrinsic curvature, $K_{i j}=\frac{1}{2} L_{n} g_{i j}$, in the gravitational case, the electric one-form is defined by

$$
E \equiv-i_{n} F
$$

where $F=d A+A \wedge A$ is the YM field strength. We also define the momentum belonging to the Higgs field $H$ by

$$
\Pi \equiv i_{n} D H
$$

where $D \equiv d+[A \mid \quad]$ denotes the covariant derivative with respect to $A$.

In terms of these quantities, the ADM decomposition of the YMH equations yields the following equations (which we list here only for vanishing shift, $\beta=0$, since they will be needed only for a static background and in a gauge with $\delta \beta=0$; the general case is obtained after the substitution $\partial_{t} \mapsto \partial_{t}-\bar{L}_{\beta}$.): The Gauss constraint is

$$
-(* D * F-J)_{0}=* \bar{D} * E-[H, \Pi] .
$$

The evolution equations comprise the definition of $E$ and II,

$$
\begin{aligned}
& E=-\frac{1}{\alpha}\left(\partial_{t} \bar{A}+\bar{D}(\alpha \Phi)\right), \\
& \Pi=\frac{1}{\alpha} \partial_{t} H-[\Phi, H],
\end{aligned}
$$

respectively, and the equations

$$
\begin{aligned}
& -\alpha(* D * F-J)^{i}=\frac{1}{\sqrt{\bar{g}}} \partial_{t}\left(\sqrt{\bar{g}} E^{i}\right)-\bar{\star} \bar{D} \bar{*}(\alpha \bar{F})^{i} \\
& -\left[\alpha \Phi, E^{i}\right]-\left[\alpha H, \bar{D}^{i} H\right], \\
& \alpha\left(* D * D H+2 V^{\prime} H\right)=\frac{1}{\sqrt{\bar{g}}} \partial_{t}(\sqrt{\bar{g}} \Pi)-\bar{*} \bar{D} \bar{*}(\alpha \bar{D} H) \\
& -[\alpha \Phi, \Pi]+2 \alpha V^{\prime} H \text {. }
\end{aligned}
$$

All quantities with a bar refer to the 3-metric $\overline{\boldsymbol{g}}$ and to the magnetic part of the potential, $\bar{A}$. Also, $E^{i} \equiv \bar{g}^{i j} E_{j}$ is raised with the 3 -metric. The Higgs potential $V$ is assumed to be a function of $|H|^{2}=\operatorname{Tr}\left(H^{2}\right)$ only, with derivative $V^{\prime}$.

There is no evolution equation for the electric potential $\Phi$, which reflects the invariance of the theory with respect to gauge transformations of the gauge potential $A$. In fact, $\Phi$ plays a similar role as the shift $\beta$ for the gravitational ADM equations. It is easy to see that the Gauss constraint propagates as a consequence of the identity $D^{2} * F=[F \mid * F]=0$ and the corresponding consistency condition $D * J=-[D H \mid * D H]-[H, D * D H]=0$ for the matter current, which holds by virtue of the Higgs equations.

In order to solve the coupled EYMH equations, the $3+1$ decomposition of the stress-energy tensor $T_{\mu \nu}=$ $T_{\mu \nu}^{(Y M)}+T_{\mu \nu}^{(H)}$ is needed:

$$
\begin{aligned}
T_{00}^{(Y M)}= & \frac{1}{4 \pi} \operatorname{Tr}\left\{\frac{1}{2} E_{k} E^{k}+\frac{1}{4} \bar{F}_{k l} \bar{F}^{k l}\right\}, \\
T_{i 0}^{(Y M)}= & \frac{1}{4 \pi} \operatorname{Tr}\left\{E^{k} \bar{F}_{k i}\right\}, \\
T_{i j}^{(Y M)}= & \frac{1}{4 \pi} \operatorname{Tr}\left\{-E_{i} E_{j}+\frac{1}{2} \bar{g}_{i j} E_{k} E^{k}\right. \\
& \left.+\bar{F}_{i k} \bar{F}_{j}^{k}-\frac{1}{4} \bar{g}_{i j} \bar{F}_{k l} \bar{F}^{k l}\right\}, \\
T_{00}^{(H)}= & \frac{1}{4 \pi} \operatorname{Tr}\left\{\frac{1}{2} \Pi^{2}+\frac{1}{2}\left(\bar{D}_{k} H\right)\left(\bar{D}^{k} H\right)\right\}+\frac{1}{4 \pi} V, \\
T_{i 0}^{(H)}= & \frac{1}{4 \pi} \operatorname{Tr}\left\{\Pi\left(\bar{D}_{i} H\right)\right\}, \\
T_{i j}^{(H)}= & \frac{1}{4 \pi} \operatorname{Tr}\left\{\left(\bar{D}_{i} H\right)\left(\bar{D}_{j} H\right)-\frac{1}{2} \bar{g}_{i j}\left(\bar{D}_{k} H\right)\left(\bar{D}^{k} H\right)\right. \\
& \left.+\frac{1}{2} \bar{g}_{i j} \Pi^{2}\right\}-\frac{1}{4 \pi} \bar{g}_{i j} V .
\end{aligned}
$$

Here, Tr denotes an Ad-invariant innerproduct on the Lie algebra. The complete set of EYMH equations in the ADM formalism is now obtained from the expressions (5), (6) and (7) for the Einstein tensor, and the definition (8) of the extrinsic curvature.

\section{B. Gauge-invariant quantities}

For a static background the slicing may be chosen such that $\boldsymbol{g}=-\alpha^{2} d t^{2}+\overline{\boldsymbol{g}}$ and $K_{i j}=0$. As a consequence, $G_{i 0}=0$, and it is consistent to set $E=0$ and $\Pi=$ 0 . Hence, we are considering a static, purely magnetic background:

$$
A=\bar{A}_{i} d x^{i}
$$

where $\bar{A}$ and $H$ do not depend on $t$. The YMH equations reduce to

$$
\begin{gathered}
\bar{*} \bar{D} \bar{*}(\alpha \bar{F})+\left[\alpha H, \bar{D}^{i} H\right]=0, \\
\bar{*} \bar{D} \bar{*}(\alpha \bar{D} H)-2 \alpha V^{\prime} H=0 .
\end{gathered}
$$

The perturbed amplitudes, $\delta \Phi, \delta E, \delta H$ and $\delta \Pi$ are subject to both coordinate and gauge transformations. Since $E$ and $\Pi$ vanish on the background, we except them to be gauge-invariant to first order: Under infinitesimal coordinate transformations generated by a vector field $X=\left(f, X^{i}\right)$, one finds, using the same formulae as in section II B:

$$
\begin{aligned}
\delta E_{i} & \mapsto \delta E_{i}+\alpha \bar{F}_{i j} f^{j}, \\
\delta \Pi & \mapsto \delta \Pi+\alpha\left(\bar{D}_{j} H\right) f^{j} .
\end{aligned}
$$


Therefore, $\delta E$ and $\delta \Pi$ are vector-invariant quantities. Under an infinitesimal gauge transformation generated by a Lie-algebra valued scalar $\chi$, we find that

$$
\delta \Phi \mapsto \delta \Phi-\frac{1}{\alpha} \dot{\chi}
$$

whereas $\delta E$ and $\delta \Pi$ remain invariant. Hence, the gaugeinvariant quantities to evolve are the perturbed electric one-form and the perturbed Higgs momentum.

\section{The linearized equations}

In order to simplify the derivation of the wave operator, it is convenient to choose the gauge function $\chi$ such that $\delta \Phi=0$. In this gauge, the linearized ADM Gauss constraint becomes

$$
-\delta(* D * F-J)_{0}=\bar{*} \bar{D} \bar{*} \delta E-[H, \delta \Pi],
$$

while the ADM evolution equations are

$$
\delta E=-\frac{1}{\alpha} \partial_{t} \delta \bar{A}, \quad \delta \Pi=\frac{1}{\alpha} \partial_{t} \delta H
$$

and

$$
\begin{aligned}
-\alpha \delta(* D * F-J)^{i}=\delta \dot{E}^{i} & -\delta \bar{*} \bar{D} \bar{*}(\alpha \bar{F})^{i}-\delta\left[\alpha H, \bar{D}^{i} H\right], \\
\alpha \delta\left(* D * D H+2 V^{\prime} H\right) & =\delta \dot{\Pi}-\delta \bar{*} \bar{D} \bar{*}(\alpha \bar{D} H) \\
& +2 \delta\left(\alpha V^{\prime} H\right)
\end{aligned}
$$

These equations have exactly the same structure as the gravitational equations (17), (18) and (19), where $\delta \dot{\bar{A}}_{i}$, $\delta \dot{H}, \delta \dot{\bar{g}}_{i j}$, as well as $\delta E_{i}, \delta \Pi, \delta K_{i j}$ correspond to each other. In order to obtain a wave equation for $\delta E$ and $\delta \Pi$, we therefore differentiate the two equations in $(43)$ with respect to $t$. Again defining vector-invariant quantities according to

$$
\mathcal{E} \equiv \alpha \delta E=-\partial_{t} \delta \bar{A}, \quad \Psi \equiv-\alpha \delta \Pi=-\partial_{t} \delta H,
$$

and also using

$$
\partial_{t} \delta \bar{g}_{i j}=2 L_{i j}, \quad \partial_{t} \delta \alpha=A,
$$

yields the following expressions:

$$
\begin{aligned}
& -\alpha \partial_{t} \delta(* D * F-J)_{i} \\
= & \frac{1}{\alpha} \ddot{\mathcal{E}}_{i}+\bar{*} \bar{D} \bar{*} \alpha \bar{D} \mathcal{E}_{i}-\alpha\left[\bar{F}_{i j}, \mathcal{E}^{j}\right]+\alpha\left[H,\left[\mathcal{E}_{i}, H\right]\right] \\
+ & \alpha \bar{D}_{i}([H, \Psi])-2 \alpha\left[\bar{D}_{i} H, \Psi\right] \\
- & 2 \bar{D}^{j}\left(L_{k j} \alpha \bar{F}_{i}^{k}\right)+2 \alpha \bar{F}^{k j} \bar{\nabla}_{j} L_{k i}-\alpha \bar{F}_{i j} \bar{\nabla}^{j}\left(L+\frac{A}{\alpha}\right),
\end{aligned}
$$

and

$$
\begin{aligned}
& -\alpha \partial_{t} \delta\left(* D * D H+2 V^{\prime} H\right) \\
= & \frac{1}{\alpha} \ddot{\Psi}-\bar{*} \bar{D} \bar{*} \alpha \bar{D} \Psi+4 \alpha V^{\prime \prime} \operatorname{Tr}(H \Psi) H+2 \alpha V^{\prime} \Psi \\
- & {\left[\bar{D}_{k}\left(\alpha \mathcal{E}^{k}\right), H\right]-2 \alpha\left[\mathcal{E}^{k}, \bar{D}_{k} H\right] } \\
- & 2 \bar{D}^{i}\left(L_{i j} \alpha \bar{D}^{j} H\right)+\alpha\left(\bar{D}_{j} H\right)\left(L+\frac{A}{\alpha}\right),
\end{aligned}
$$

for the linearized YM equations and the linearized Higgs equations, respectively. Here, we have also used the background equations (42). Equations (44) and (45) provide a system of evolution equations for $\mathcal{E}$ and $\Psi$, which is manifestly symmetric with respect to the inner product

$$
\begin{aligned}
& \left\langle\left(\mathcal{E}^{(1)}, \Psi^{(1)}\right),\left(\mathcal{E}^{(2)}, \Psi^{(2)}\right)\right\rangle \\
& \equiv \int_{\Sigma} \operatorname{Tr}\left\{\bar{g}^{i j} \mathcal{E}_{i}^{(1)} \mathcal{E}_{j}^{(2)}+\Psi^{(1)} \Psi^{(2)}\right\} \bar{\eta} .
\end{aligned}
$$

However, we are again faced with the problem that the evolution equation governing $\mathcal{E}$ is not hyperbolic. Like in the gravitational case, a hyperbolic wave operator can be constructed without loosing the symmetry. Again, the strategy is to add suitable combinations of the linearized Gauss constraint,

$$
-\alpha \delta(* D * F-J)_{0}=\alpha \bar{*} \bar{D} \bar{*}\left(\frac{\mathcal{E}}{\alpha}\right)+[H, \Psi],
$$

and its spatial derivatives. Furthermore, one can use the linearized momentum constraint,

$$
\begin{aligned}
C_{i} & \equiv \alpha^{2} \delta\left(G_{i 0}-8 \pi G T_{i 0}\right) \\
& =\alpha^{2} \bar{G}_{i j k l} \bar{\nabla}^{j}\left(\frac{L^{k l}}{\alpha}\right)-2 G \alpha \operatorname{Tr}\left(\bar{F}_{i}^{k} \mathcal{E}_{k}-\left(\bar{D}_{i} H\right) \Psi\right),
\end{aligned}
$$

in order to eliminate the divergence terms of $L_{i j}$ in the last lines of Eqs. (44) and (45). The motivation for this (besides eliminating first order derivatives) is the following: We will see that the adjoint of the operator governing the matter perturbation in the linearized stress-energy tensor contains no divergence term. In order to formulate the linearized EYMH equations as a symmetric wave equation, no divergences of $L_{i j}$ must therefore appear in the YMH equations. The correct combinations turn out to be

$$
\begin{aligned}
\Lambda_{i}^{(Y M)} & \equiv-\alpha \partial_{t} \delta(* D * F-J)_{i} \\
& +\frac{1}{\alpha} \bar{D} \alpha^{3} \delta(* D * F-J)_{0}+2 \bar{F}_{i}^{k} C_{k}, \\
\Lambda^{(H)} & \equiv-\alpha \partial_{t} \delta\left(* D * D H+2 V^{\prime} H\right) \\
& -\alpha^{2}\left[\delta(* D * F-J)_{0}, H\right]+2\left(\bar{D}^{k} H\right) C_{k} .
\end{aligned}
$$

This yields the following hyperbolic, symmetric equations:

$$
\begin{aligned}
\Lambda_{i}^{(Y M)} & =\square \mathcal{E}_{i}-\alpha\left[\bar{F}_{i j}, \mathcal{E}^{j}\right]+\alpha\left[H,\left[\mathcal{E}_{i}, H\right]\right] \\
& +4 G \alpha \operatorname{Tr}\left(\bar{F}_{k}^{l} \mathcal{E}_{l}-\bar{D}_{k} H \Psi\right) \bar{F}_{i}{ }^{k}-2\left[\bar{D}_{i}(\alpha H), \Psi\right]
\end{aligned}
$$




$$
\begin{aligned}
& +2 \alpha \bar{F}^{j k} \bar{\nabla}_{k} L_{i j}-\frac{2}{\alpha} L_{k j} \bar{D}^{j}\left(\alpha^{2} \bar{F}^{k i}\right) \\
& +\alpha \bar{F}_{i j} \bar{\nabla}^{j}\left(L-\frac{A}{\alpha}\right)+2 \alpha^{k} \bar{F}_{k i} L, \\
\Lambda^{(H)}= & \square \Psi+4 \alpha V^{\prime \prime} \operatorname{Tr}(H \Psi) H+2 \alpha V^{\prime} \Psi+\alpha[[H, \Psi], H] \\
- & 4 G \alpha \operatorname{Tr}\left(\bar{F}_{k}^{l} \mathcal{E}_{l}-\bar{D}_{k} H \Psi\right) \bar{D}^{k} H-2\left[\mathcal{E}^{k}, \bar{D}_{k}(\alpha H)\right] \\
- & \frac{2}{\alpha} L_{j k} \bar{D}^{j}\left(\alpha^{2} \bar{D}^{k} H\right) \\
- & \alpha\left(\bar{D}_{j} H\right) \bar{\nabla}^{j}\left(L-\frac{A}{\alpha}\right)+2 \alpha^{k}\left(\bar{D}_{k} H\right) L,
\end{aligned}
$$

where the d'Alembertians are defined by

$$
\begin{aligned}
& \square \mathcal{E}_{i}=\frac{1}{\alpha} \partial_{t}^{2} \mathcal{E}_{i}+\bar{D}^{\dagger} \alpha \bar{D} \mathcal{E}_{i}+\frac{1}{\alpha} \bar{D} \alpha^{3} \bar{D}^{\dagger}\left(\frac{\mathcal{E}_{i}}{\alpha}\right), \\
& \square \Psi=\frac{1}{\alpha} \partial_{t}^{2} \Psi+\bar{D}^{\dagger} \alpha \bar{D} \Psi,
\end{aligned}
$$

and where - for Lie-algebra valued $p$-forms $\omega$ on $\bar{M}-$ the operator adjoint of $\bar{D}$ is $\bar{D}^{\dagger} \omega=(-1)^{p} \bar{*} \bar{\nabla} \bar{*} \omega$. In order to complete the pulsation equations, we still have to compute the linearized stress-energy tensor: Using the background equations and

$$
\delta \dot{\bar{F}}_{i j}=-\bar{D}_{i} \mathcal{E}_{j}+\bar{D}_{j} \mathcal{E}_{i}, \quad \delta \dot{H}=-\Psi, \quad \text { etc... }
$$

one finds that the expression for $\Lambda_{i j}$ - defined in (26) with $G_{\mu \nu}$ replaced by $G_{\mu \nu}-8 \pi G T_{\mu \nu}$ - is given by

$$
\begin{aligned}
\Lambda_{i j} & =\Lambda_{i j}^{V}-4 G \operatorname{Tr}\left\{\bar{D}_{k}\left(\alpha \bar{F}_{(i}{ }^{k} \mathcal{E}_{j)}\right)+\frac{1}{\alpha} \mathcal{E}_{k} \bar{D}_{(i}\left(\alpha^{2} \bar{F}_{j)}^{k}\right)\right. \\
& \left.-\bar{g}_{i j} \mathcal{E}^{l} \alpha^{k} \bar{F}_{k l}+\frac{1}{\alpha} \bar{D}_{(i}\left(\alpha^{2} \bar{D}_{j)} H\right) \Psi+\bar{g}_{i j} \alpha^{k}\left(\bar{D}_{k} H\right) \Psi\right\} \\
& +4 G \alpha \operatorname{Tr}\left\{\bar{F}_{i}^{k} \bar{F}_{j}^{l} L_{k l}-\frac{1}{4} \bar{F}_{k l} \bar{F}^{k l} L_{i j}-\frac{1}{8} \bar{g}_{i j} \bar{F}_{k l} \bar{F}^{k l} L\right\} \\
& -4 G \alpha \Phi L_{i j}+2 \bar{g}_{i j} \alpha \Phi L,
\end{aligned}
$$

where $\Lambda_{i j}^{V}$ is defined in Eq. (27). The matter perturbations arising in $\Lambda_{i j}$ perfectly fit together with the gravitational perturbations arising in $\Lambda^{(Y M)}$ and $\Lambda^{(H)}$, with exception of the terms involving $\left(L-\frac{A}{\alpha}\right)$. Hence, one either has to adopt the harmonic gauge, $A=\alpha L$, for which the operator is symmetric but the kinetic energy is not definite (see section II ), or the maximal slicing condition, $L=0$, for which the operator acting on $L_{i j}$ is hyperbolic and symmetric, but the perturbed lapse is still present. As in the gravitational case, we adopt the maximal slicing condition.

\section{Summary}

The linearized EYMH equations (with arbitrary compact gauge group) for a static, purely magnetic background, yield a hyperbolic, formally self-adjoint operator for the variation of the extrinsic curvature, the electric one-form and the Higgs momentum. All these quantities are invariant with respect to both infinitesimal gauge transformations of the gauge fields and infinitesimal coordinate transformations within the slices $\Sigma_{t}$. The wave operator is constructed from appropriate combinations of the linearized ADM equations, as defined in Eqs. (26) and (49). In the maximal slicing gauge, the evolution equations are described by the trace-less part of the tensor $\Lambda_{i j}$, given in Eq. (52), as well as the expressions for $\Lambda^{(Y M)}$ and $\Lambda^{(H)}$ defined in Eqs. (50) and (51), respectively, where one sets $L=0$. The constraint equations are the momentum constraint, (48) and the Gauss constraint, (47). (Additional constraints involving also perturbations of the metric, the gauge potential and the Higgs field themselves are the Hamilton constraint and all evolution equations, which were differentiated with respect to time in order to construct the wave operator.) Furthermore, the trace of the tensor $\Lambda_{i j}$ yields the following elliptic equation for $A$ :

$$
\begin{aligned}
& \left(\bar{\Delta}-R_{00}\right) A=2 \bar{\nabla}^{k}\left\{\alpha^{l} L_{k l}+G \operatorname{Tr}\left(\alpha \bar{F}_{l k} \mathcal{E}^{l}\right)\right\} \\
& \quad+2 G \alpha \operatorname{Tr}\left\{\left[H, \bar{D}^{k} H\right] \mathcal{E}_{k}+2 V^{\prime} H \Psi-\bar{F}^{k m} \bar{F}_{m}^{l} L_{k l}\right\},
\end{aligned}
$$

where the momentum constraint and the background equations have been used as well. At least in the pure EYM case, this equation is always solvable, since either $2 R_{00}=G \operatorname{Tr}\left(\bar{F}_{k l} \bar{F}^{k l}\right)>0$ and the operator on the left is invertible or the magnetic field vanishes and the equation reduces to its vacuum counterpart (37). In the presence of Higgs fields further investigations are needed, since $R_{00}^{(H)}=-2 G V$ is negative in that case (i.e. the strong energy condition does not hold for the Higgs part of the stress-energy tensor). In some relevant situations, however, the trace equation causes no problems: In particular, this equation is void for odd-parity perturbations of a spherically symmetric background.

Finally, it is also instructive to compute the differentiated Hamilton constraint, defined as in (35) - with $G_{\mu \nu}$ replaced by $G_{\mu \nu}-8 \pi G T_{\mu \nu}$. This yields $\alpha \Lambda_{00}=$ $-\bar{\nabla}^{j} C_{j}$, where $C_{j}$ are the constraint variables defined in Eq. (48). The system of equations defined by $\left(\Lambda_{00}, \hat{\Lambda}_{i j}, \Lambda_{i}^{(Y M)}, \Lambda^{(H)}\right)$ is symmetric in the amplitudes $u \equiv\left(A, L_{i j}, \mathcal{E}_{i}, \Psi\right)$ with respect to the inner product

$$
\begin{aligned}
\left\langle u^{(1)}, u^{(2)}\right\rangle & \equiv \int_{\Sigma}\left[A^{(1)} A^{(2)}+\bar{g}^{i k} \bar{g}^{j l} L_{i j}^{(1)} L_{k l}^{(2)}+\right. \\
& \left.+2 G \operatorname{Tr}\left\{\bar{g}^{i j} \mathcal{E}_{i}^{(1)} \mathcal{E}_{j}^{(2)}+\Psi^{(1)} \Psi^{(2)}\right\}\right] \bar{\eta} .
\end{aligned}
$$

As $\Lambda_{00}=0$ holds by virtue of the momentum constraint, this again implies that all terms involving the amplitude $A$ in the remaining equations $\hat{\Lambda}_{i j}, \Lambda_{i}^{(Y M)}$ and $\Lambda^{(H)}$ are orthogonal to the (momentum-) constraint manifold. Therefore, the variation of the lapse does not appear if the evolution equations are projected on the constraint manifold. The initial value problem is solved similarly to the vacuum case, see section IIIA. 


\section{THE COUPLING TO PERFECT FLUIDS}

At a first glance, it may be surprising that our formulation also applies to perfect fluids. It is, however, known 21] that Lagrangian formulations exist in this case. As we show in Appendix B, the linear fluctuations of a selfgravitating perfect fluid are described by the linearized metric and the Lagrangian displacement vector. If the background is static, it will be shown that the wave operator acts on the components of the extrinsic curvature and the time-derivative of the displacement vector.

\section{A. The ADM equations}

The stress-energy tensor for a perfect fluid is given by

$$
T_{\mu \nu}=(\rho+P) u_{\mu} u_{\nu}+P g_{\mu \nu},
$$

where $u$ is a time-like vector field, describing the fluid's motion, normalized such that $\boldsymbol{g}(u, u)=-1$. The fields $\rho$ and $P$ denote the energy density and the pressure of the fluid, respectively. The ADM split of $u$ is given by

$$
u=\gamma\left(e_{0}+v^{i} e_{i}\right)
$$

where $\gamma^{-2}=1-\overline{\boldsymbol{g}}(v, v)$ and $e_{0}$ is the previously introduced normal unit vector field orthogonal to $\Sigma_{t}$. We choose the sign of $\gamma$ such that the fluid flows in the same direction as $e_{0}$.

The relativistic Euler equations are obtained from $\nabla^{\nu} T_{\mu \nu}=0$ :

$$
\begin{aligned}
& 0=u^{\mu} \nabla^{\nu} T_{\mu \nu}=(\rho+P) \theta+\nabla_{u} \rho, \\
& 0=q_{\mu}^{\alpha} \nabla^{\nu} T_{\alpha \nu}=(\rho+P) a_{\mu}+q_{\mu}^{\alpha} \nabla_{\alpha} P,
\end{aligned}
$$

where $\theta=\nabla_{\mu} u^{\mu}$ is the expansion, $a_{\mu}=\left(\nabla_{u} u\right)_{\mu}=$ $u^{\nu} \nabla_{\nu} u_{\mu}$ is the acceleration vector, and where $q_{\beta}^{\alpha}=$ $\delta_{\beta}^{\alpha}+u^{\alpha} u_{\beta}$ projects onto the spaces orthogonal to $u$. Inserting the expression (54) above yields

$$
\begin{aligned}
\theta & =\frac{1}{\alpha} \dot{\gamma}+\gamma K+\frac{1}{\alpha} \bar{\nabla}^{j}\left(\alpha u_{j}\right), \\
a_{i} & =\frac{\gamma}{\alpha}\left(\dot{u}_{i}+\gamma \alpha_{i}\right)+u^{j} \bar{\nabla}_{j} u_{i} .
\end{aligned}
$$

Here, we have assumed that the shift vector $\beta$ vanishes, such that $u_{i}=\gamma v^{i}$. For linear perturbations of a static background there is no loss of generality in doing so since we will choose a gauge with $\delta \beta=0$. The stress-energy tensor gives

$$
\begin{aligned}
T_{00} & =\gamma^{2}(\rho+P) \\
T_{i 0} & =-\gamma(\rho+P) u_{i} \\
T_{i j} & =(\rho+P) u_{i} u_{j}+P \bar{g}_{i j} .
\end{aligned}
$$

In order to get a closed system of equations, an equation of state is needed as well. In the following we will only assume that this has the form $P=P(\rho)$.

\section{B. Coordinate-invariant quantities}

For a static background one has $G_{i 0}=0$, and it is consistent to set $u_{i}=0$ (i.e. the flow is static). The $\mathrm{ADM}$ equations then reduce to

$$
\begin{aligned}
& 0=\frac{1}{\alpha} \bar{\Delta} \alpha-4 \pi G(\rho+3 P), \\
& 0=\bar{R}_{i j}-\frac{1}{\alpha} \bar{\nabla}_{i} \bar{\nabla}_{j} \alpha-4 \pi G \bar{g} i j(\rho-P), \\
& 0=\bar{\nabla}_{j} P+(\rho+P) \frac{\alpha_{j}}{\alpha} .
\end{aligned}
$$

Under an infinitesimal coordinate transformation generated by $\left(X^{\mu}\right)=\left(f, X^{i}\right)$ one has the following behavior:

$$
\begin{aligned}
\delta u_{i} & \mapsto \delta u_{i}-\alpha f_{i}, \\
\delta \rho & \mapsto \delta \rho+X^{s} \bar{\nabla}_{s} \rho, \\
\delta P & \mapsto \delta P+X^{s} \bar{\nabla}_{s} P .
\end{aligned}
$$

Therefore, $\delta u_{i}$ is a vector-invariant quantity, and using (13), we may also construct the vector-invariant amplitudes

$$
\begin{aligned}
\delta \dot{\rho}^{(i n v)} & \equiv \delta \dot{\rho}-\delta \beta^{s} \bar{\nabla}_{s} \rho, \\
\delta \dot{P}^{(i n v)} & \equiv \delta \dot{P}-\delta \beta^{s} \bar{\nabla}_{s} P .
\end{aligned}
$$

Using the transformation properties of $\delta u_{i}$ it is tempting to construct full coordinate-invariant amplitudes. These correspond to Lagrangian deformations of $\boldsymbol{g}, \rho$ and $P$ (see Appendix B). Moreover, it turns out that using the relations $(\mathrm{B} 5)$, one can find a wave equation for $\Delta \ddot{\bar{g}}_{i j}$ alone. Unfortunately, the resulting pulsation equation is not symmetric.

The correct quantities to evolve - from the philosophy adopted in this paper - are the vector-invariant quantities $\delta K_{i j}$ and $\delta u_{i}$. This is motivated by the fact that for a static background, the second relation in (B5) yields $\alpha \delta u_{i}=\dot{\xi}_{i}$.

\section{The linearized equations}

We start with the linearization of the relativistic Euler equations (55) and (56). As before, we make use of the gauge freedom in order to set $\delta \beta=0$. Noting that $\gamma$ is quadratic in $u_{i}$ and that $u_{i}$ vanishes on the background, we obtain

$$
\begin{aligned}
\delta \theta & =\delta K+\frac{1}{\alpha} \bar{\nabla}^{j}\left(\alpha \delta u_{j}\right), \\
\delta a_{i} & =\frac{1}{\alpha} \delta \dot{u}_{i}+\bar{\nabla}_{i}\left(\frac{\delta \alpha}{\alpha}\right) .
\end{aligned}
$$

Also using the background equation (60), the linearization of equation (5.5) gives

$$
\delta \dot{\rho}=-\frac{1}{\alpha}\left(\alpha^{2}(\rho+P) \delta K+\bar{\nabla}^{k}\left[\alpha^{2}(\rho+P) \delta u_{k}\right]\right) .
$$


Defining the speed of sound $c_{s}$ by $c_{s}^{2}=\partial P / \partial \rho$, we also have

$$
\delta \dot{P}=-\frac{c_{s}^{2}}{\alpha}\left(\alpha^{2}(\rho+P) \delta K+\bar{\nabla}^{k}\left[\alpha^{2}(\rho+P) \delta u_{k}\right]\right) .
$$

[The above equations also follow from the relations (B5) derived in Appendix B.] Finally, using the fact that $P$ is a function of $\rho$ only, the linearization of equation (56) yields

$$
\delta\left(q_{\mu}^{\alpha} \nabla^{\nu} T_{\alpha \nu}\right)=\frac{1}{\alpha} \delta \dot{u}_{i}+\bar{\nabla}_{i}\left(\frac{\delta \alpha}{\alpha}+\frac{\delta P}{\rho+P}\right) .
$$

An evolution equation for $\delta u_{i}$ is obtained after differentiating this equation with respect to $t$ and eliminating $\delta \dot{P}$ using equation (62). Defining $B=\alpha^{3}(\rho+P)$, $C=\alpha^{2} c_{s}^{2} / B$,

$$
W_{i} \equiv \alpha^{2}(\rho+P) \delta u_{j}
$$

and recalling that $L=\alpha \delta K, A=\delta \dot{\alpha}$, we find

$$
\begin{aligned}
\partial_{t} \delta\left(q_{i}^{\alpha} \nabla^{\nu} T_{\alpha \nu}\right) & =\frac{1}{B} \partial_{t}^{2} W_{i}-\bar{\nabla}_{i}\left(C \bar{\nabla}^{k} W_{k}\right) \\
& +\bar{\nabla}_{i}\left(\frac{A}{\alpha}-c_{s}^{2} L\right)=0 .
\end{aligned}
$$

Furthermore, equation (63) yields the following circularity condition:

$$
\bar{\nabla}_{i}\left(\frac{\dot{W}_{j}}{B}\right)-\bar{\nabla}_{j}\left(\frac{\dot{W}_{i}}{B}\right)=0 .
$$

This condition would also allow to introduce a potential $\Phi$, defined by $W_{i}=B \bar{\nabla}_{i} \Phi$. However, in order to obtain symmetric equations, it turns out to be necessary to formulate the pulsation equations in terms of $\delta u_{i}$ rather than $\Phi$. The condition $(66)$ is, therefore, kept as a constraint equation for the system. That this constraint propagates is obvious from Eq. (65). The circularity condition is used to cast the evolution equation into a hyperbolic and symmetric form. Indeed, taking a time derivative and using Eq. (66), one can rewrite Eq. (65) in a manifestly hyperbolic form without destroying the symmetry:

$$
\begin{aligned}
0 & =\frac{1}{B} \partial_{t}^{2} \dot{W}_{i}-\bar{\nabla}^{k} C \bar{\nabla}_{k} \dot{W}_{i}+F^{k} \bar{\nabla}_{i} \dot{W}_{k}-\bar{\nabla}^{k}\left[F_{i} \dot{W}_{k}\right] \\
& +\frac{1}{B} \bar{\nabla}^{k}\left(C B_{k}\right) \dot{W}_{i}+\left(\bar{\nabla}_{i} \bar{\nabla}_{k} C\right) \dot{W}^{k}-\frac{C}{B^{2}} B_{i} B_{k} \dot{W}^{k} \\
& +C \bar{R}_{i k} \dot{W}^{k}+\bar{\nabla}_{i}\left(\frac{\dot{A}}{\alpha}-c_{s}^{2} \dot{L}\right),
\end{aligned}
$$

where $F_{k}=\bar{\nabla}_{k}(B C) / B$.

In order to complete the pulsation equations, we have to compute the linearized stress-energy tensor. Using (57) and (62) in the expression for $\Lambda_{i j}$ - defined in (26) with $G_{\mu \nu}$ replaced by $G_{\mu \nu}-8 \pi G T_{\mu \nu}$ - we find

$$
\begin{aligned}
\Lambda_{i j} & =\Lambda_{i j}^{V}-8 \pi G\left(\alpha(\rho-P) L_{i j}\right. \\
& +\frac{2}{\alpha} \bar{G}_{i j k l} \bar{\nabla}^{k}\left(\alpha W^{l}\right)+\left(1-c_{s}^{2}\right) \bar{g}_{i j} \bar{\nabla}^{k} W_{k} \\
& \left.-\alpha \bar{g}_{i j}\left[c_{s}^{2}(\rho+P)-(\rho-P)\right] L\right),
\end{aligned}
$$

where $\Lambda_{i j}^{V}$ is defined in (27). In order to obtain symmetric equations, we use the linearized momentum constraint,

$$
C_{i}=\alpha^{2} \delta\left(G_{i 0}-8 \pi G T_{i 0}\right)=\alpha^{2} \bar{G}_{i j k l} \bar{\nabla}^{j}\left(\frac{L^{k l}}{\alpha}\right)+8 \pi G W_{i} .
$$

Defining

$$
\Lambda^{(F)} \equiv \partial_{t} \delta\left(q_{i}^{\alpha} \nabla^{\nu} T_{\alpha \nu}\right)+\frac{2}{\alpha} C_{i},
$$

we finally obtain

$$
\begin{aligned}
\Lambda^{(F)} & =\frac{1}{B} \partial_{t}^{2} W_{i}-\bar{\nabla}_{i}\left(C \bar{\nabla}^{k} W_{k}\right)+\frac{16 \pi G}{\alpha} W_{i} \\
& +2 \alpha \bar{G}_{i j k l} \bar{\nabla}^{j}\left(\frac{L^{k l}}{\alpha}\right)+\bar{\nabla}_{i}\left(\left(1-c_{s}^{2}\right) L-L+\frac{A}{\alpha}\right) .
\end{aligned}
$$

This shows that, with the exception of the last term involving $\left(L-\frac{A}{\alpha}\right)$, the matter perturbations arising in $\Lambda_{i j}$ perfectly fit together with the gravitational perturbations arising in $\Lambda^{(F)}$.

Like in the vacuum case, we now adopt the maximal slicing gauge $L=0$. The evolution equations for $L_{i j}$ and $W_{i}$ are given by the trace-less part of $\Lambda_{i j}$ and $\Lambda^{(F)}$, where one sets $L=0$. The constraint equations comprise the linearized momentum constraint, (69), and the circularity condition, (66). The trace part of $\Lambda_{i j}$ yields the following equation for $A$ :

$$
\left(\bar{\Delta}-R_{00}\right) A=2 \bar{\nabla}^{k}\left(\alpha^{l} L_{k l}\right)-4 \pi G\left(1+3 c_{s}^{2}\right) \bar{\nabla}^{k} W_{k},
$$

with $R_{00}=4 \pi G(\rho+3 P)$. Here, the momentum constraint has been used in order to simplify the equation. As long as $\rho+3 P$ is positive, the operator on the LHS is invertible and thus the equation for $A$ is solvable. The differentiated Hamilton constraint - defined in Eq. (35) with $G_{\mu \nu}$ replaced by $G_{\mu \nu}-8 \pi G T_{\mu \nu}$ - yields

$$
\Lambda_{00}=-\frac{1}{\alpha} \bar{\nabla}^{i}\left\{\alpha^{2} \bar{\nabla}^{j}\left(\frac{L_{i j}}{\alpha}\right)+8 \pi G W_{i}\right\} .
$$

The system of equations defined by $\left(\Lambda_{00}, \hat{\Lambda}_{i j}, \Lambda^{(F)}\right)$ is symmetric in the amplitudes $u \equiv\left(A, L_{i j}, W_{i}\right)$ with respect to the inner product

$$
\begin{aligned}
& \left\langle u^{(1)}, u^{(2)}\right\rangle \\
& \equiv \int_{\Sigma}\left[A^{(1)} A^{(2)}+\bar{g}^{i k} \bar{g}^{j l} L_{i j}^{(1)} L_{k l}^{(2)}+8 \pi G \bar{g}^{i j} W_{i}^{(1)} W_{j}^{(2)}\right] \bar{\eta} .
\end{aligned}
$$

As before, $\Lambda_{00}=0$ is satisfied by virtue of the momentum constraint and, as a consequence, the variation of the 
lapse does not appear if the wave operator is projected onto the constraint manifold.

Finally, the evolution equations $\hat{\Lambda}_{i j}=0$ and $\Lambda^{(F)}=$ 0 can be written in a manifestly hyperbolic form by a further differentiation with respect to $t$, as we have shown above. The initial value problem is solved similarly to the vacuum case.

\section{CONCLUSION}

We have shown that the perturbation equations governing linear fluctuations on a static background can be cast into the form of a constraint symmetric wave equation for gauge-invariant quantities. In particular, we have discussed the initial value formulation for vacuum fluctuations of vacuum space-times, where the adapted gaugeinvariant quantities are the components of the linearized extrinsic curvature tensor. For a spherically symmetric background, the constraint equations have been eliminated in a natural way, and the equations of ReggeWheeler and Zerilli were rederived. An important new feature of the curvature-based perturbation formalism presented in this paper is that it admits a natural generalization to gravitating matter fields.

As a first example, the pulsation operator governing fluctuations on a static, purely magnetic EYMH configuration were derived for an arbitrary compact gauge group. These pulsation equations are expected to be valuable in order to discuss the stability of spherically symmetric solutions to the EYMH equations with respect to non-spherical symmetric perturbations. In a forthcoming article [13], we will show that the BartnikMcKinnon solitons 14 and the corresponding black holes with hair 15 admit no unstable modes in the odd-parity sector with total angular momentum $\ell \geq 1$. It should also be interesting to generalize the investigations to solutions with Higgs fields or with a negative cosmological constant 16], since some of these solutions are known to be linearly stable with respect to radial perturbations. Also, the stability of new static, axially symmetric configurations 17] can be discussed within the new framework.

In a second example, we have shown that a symmetric formulation of the equations governing linear fluctuation of a self-gravitating perfect fluid exists. Provided that the constraint and the dynamical variables can be decoupled in a "symmetric way", the resulting equations are expected to be useful in order to study the stability of static, relativistic stars, or the emission of gravitational waves from pulsating neutron stars [18], [19]. In order to discuss the fluctuations of rapidly rotating configurations, it is our aim to generalize the curvature-based approach to general stationary, i.e. non-static, background configurations. However, this requires further investigations, since the derivation of the wave operator must be generalized to cases where the extrinsic curvature of the background no longer vanishes.
Finally, we mention that the symmetric formulation could also be useful for second (or higher) order perturbation theory, since the $n$ 'th order perturbation is governed by the same wave operator as the first oder perturbation, but with a source term depending on perturbations up to order $n-1$.

\section{Acknowledgements}

O.S. would like to thank D. Giulini for many helpful discussions. This work was in parts supported by the Swiss National Science Foundation.

\section{APPENDIX A: DERIVATION OF THE REGGE-WHEELER AND ZERILLI EQUATIONS}

In this Appendix, we specialize the general formalism to a static spherically symmetric background and show how to project the wave operator onto the momentum constraint manifold. This will result in a new - and transparent - derivation of the Regge-Wheeler and the Zerilli equations.

A convenient parametrization of the spherically symmetric 3-metric is

$$
\overline{\boldsymbol{g}}=d x^{2}+r^{2} \hat{\boldsymbol{g}},
$$

where $x$ is a radial coordinate and where $\hat{\boldsymbol{g}}=d \Omega^{2}$ is the standard volume element on $S^{2}$. The field $r$ and the lapse $\alpha$ are functions of $x$ only. The Christoffel symbols with respect to $\overline{\boldsymbol{g}}$ become

$$
\begin{aligned}
& \bar{\Gamma}_{x x}^{x}=\bar{\Gamma}_{x B}^{x}=0, \quad \bar{\Gamma}_{A B}^{x}=-r r^{\prime} \hat{g}_{A B}, \\
& \bar{\Gamma}_{x x}^{C}=0, \quad \bar{\Gamma}_{x B}^{C}=\frac{r^{\prime}}{r} \delta_{B}^{C}, \quad \bar{\Gamma}_{A B}^{C}=\hat{\Gamma}_{A B}^{C},
\end{aligned}
$$

where capital indices refer to coordinates on the 2-sphere, and a prime denotes differentiation with respect to $x$. The components of the Riemann tensor are given by

$$
\begin{aligned}
& \bar{R}_{A x B}^{x}=-r r^{\prime \prime} \hat{g}_{A B}, \quad \bar{R}_{C A B}^{x}=0, \\
& \bar{R}_{C A B}^{D}=2\left(1-r^{2}\right) \delta_{[A}^{D} \hat{g}_{B] C} .
\end{aligned}
$$

Einstein's background equations (11) become

$$
\begin{aligned}
& 0=R_{00}=\frac{\left(r^{2} \alpha^{\prime}\right)^{\prime}}{r^{2} \alpha} \\
& 0=R_{x x}=-2 \frac{r^{\prime \prime}}{r}-\frac{\alpha^{\prime \prime}}{\alpha}, \\
& 0=R_{A B}=\left(1-r^{\prime 2}-r r^{\prime \prime}-r r^{\prime} \frac{\alpha^{\prime}}{\alpha}\right) \hat{g}_{A B} .
\end{aligned}
$$

The solutions to these equations are, of course, the Schwarzschild solutions

$$
\alpha^{2}=1-\frac{2 G m}{r}, \quad \overline{\boldsymbol{g}}=\frac{d r^{2}}{\alpha^{2}}+r^{2} d \Omega^{2},
$$

where $m$ is constant. Our aim is to write the wave operator $\hat{\Lambda}_{i j}$ in terms of the background metric (A1). Since the 
background is spherically symmetric and invariant under parity reflection, $\underline{x} \mapsto-\underline{x}$, we can perform a multipole decomposition in the odd- and the even-parity sectors separately. As this decomposition can be done in an orthonormal manner, the resulting wave equations will be symmetric again.

\section{The odd-parity sector}

The symmetric tensor field $L_{i j}$ can be expanded in terms of spherical tensor harmonics. In Appendix D of Ref. [3] we have shown how these tensor harmonics can be obtained from the standard scalar spherical harmonics $Y^{\ell m}$. Since the background is spherically symmetric, perturbations belonging to different $\ell$ and $m$ decouple; in the following we shall therefore suppress these indices.

In the odd-parity sector, the expansion is given by

$$
\begin{aligned}
& L_{x x}=0, \\
& L_{x B}=n_{1} u_{1} S_{B}, \\
& L_{A B}=n_{2} r u_{2} 2 \hat{\nabla}_{(A} S_{B)},
\end{aligned}
$$

where $S_{B}=(\hat{*} d Y)_{B}$ denote the transverse spherical vector harmonics. The functions $u_{1}$ and $u_{2}$ depend on $x$ only, and the normalization constants $n_{1}$ and $n_{2}$ are chosen such that

$$
\left\langle L_{i j}, L_{i j}\right\rangle=\int_{0}^{\infty}\left(u_{1}^{2}+u_{2}^{2}\right) d x
$$

where $\langle.,$.$\rangle denotes the inner product (24). One finds$

$$
n_{1}=[2 \ell(\ell+1)]^{-1 / 2}, \quad n_{2}=[2 \ell(\ell+1) \lambda]^{-1 / 2},
$$

where we have defined $\lambda=(\ell-1)(\ell+2)$. Since the perturbation of all scalar quantities vanishes in the oddparity case, $u_{1}$ and $u_{2}$ are coordinate-invariant and the perturbation of the lapse vanishes. As a consequence, we obtain a symmetric wave equation for the amplitudes $u_{1}$ and $u_{2}$. Introducing $\mathrm{A} 4$ into the wave equation $\hat{\Lambda}_{i j}=0$ defined as in (34) gives

$$
\left(\partial_{t}^{2}-\partial_{\rho}^{2}+\boldsymbol{S}+2 V_{B G}\right) u=0
$$

where

$$
\boldsymbol{S}=\left(\begin{array}{ll}
\frac{r}{\gamma}\left(\frac{\gamma}{r}\right)_{\rho \rho}+\lambda \gamma^{2} & 2 \sqrt{\lambda} \gamma_{\rho} \\
2 \sqrt{\lambda} \gamma_{\rho} & \frac{r_{\rho \rho}}{r}+\lambda \gamma^{2}
\end{array}\right)
$$

Here, we have defined $\gamma=\alpha / r$ and the new radial coordinate $\rho$ according to $d x=\alpha d \rho$. Furthermore, $V_{B G} \equiv \gamma^{2}\left(1-r^{\prime 2}-r r^{\prime \prime}-r r^{\prime} \alpha^{\prime} / \alpha\right)$ vanishes by virtue of the background equations (A3). The momentum constraint equation yields $0=C_{B}=n_{1} u_{c} S_{B}$, where

$$
u_{c}=\frac{\gamma}{r}\left(\frac{r}{\gamma} u_{1}\right)_{\rho}-\sqrt{\lambda} \gamma u_{2}
$$

parametrizes the constraint variable.

Our aim is to find a new variable $u_{p}$, representing the dynamical variable, such that the wave equation assumes the form

$$
\left[\partial_{t}^{2}-\partial_{\rho}^{2}+\left(\begin{array}{cc}
V_{c} & 0 \\
V_{p c} & V_{p}
\end{array}\right)\right]\left(\begin{array}{l}
u_{c} \\
u_{p}
\end{array}\right)=0 .
$$

On the constraint manifold, $u_{c}=0$, one then has

$$
\left[\partial_{t}^{2}-\partial_{\rho}^{2}+V_{p}\right] u_{p}=0
$$

which is a symmetric wave equation for the dynamical variable $u_{p}$. It turns out that this can be achieved with the ansatz

$$
\left(\begin{array}{l}
u_{c} \\
u_{p}
\end{array}\right)=\boldsymbol{B}\left(\begin{array}{l}
u_{1} \\
u_{2}
\end{array}\right), \quad \boldsymbol{B}=\partial_{\rho}+\left(\begin{array}{cc}
\frac{\gamma}{r}\left(\frac{r}{\gamma}\right)_{\rho} & -\sqrt{\lambda} \gamma \\
-\sqrt{\lambda} \gamma & A
\end{array}\right),
$$

where the first row of the matrix has been chosen such that Eq. (A6) holds, while the second row has been chosen such that the matrix is symmetric. This guarantees that no first order derivatives appear in $\boldsymbol{B}^{\dagger} \boldsymbol{B}$. The key observation is that the function $A$ can be chosen such that

$$
-\partial_{\rho}^{2}+\boldsymbol{S}=\boldsymbol{B}^{\dagger} \boldsymbol{B}
$$

This is indeed the case if $A=-\frac{r_{\rho}}{r}$. As a consequence, the desired wave equation (A7) is obtained after applying $\boldsymbol{B}$ to the left of the original wave equation (A5). This yields

$$
\left[\partial_{t}^{2}+\boldsymbol{B} \boldsymbol{B}^{\dagger}\right]\left(\begin{array}{l}
u_{c} \\
u_{p}
\end{array}\right)=0
$$

where

$$
\boldsymbol{B B}^{\dagger}=-\partial_{\rho}^{2}+\left(\begin{array}{cc}
\frac{\gamma}{r}\left(\frac{r}{\gamma}\right)_{\rho \rho}+\gamma^{2} \lambda & 0 \\
0 & r\left(\frac{1}{r}\right)_{\rho \rho}+\gamma^{2} \lambda
\end{array}\right) .
$$

Thus, the constraint variables decouple from the dynamical variables, and the wave equation governing the dynamical degree of freedom is exactly the Regge-Wheeler equation [8]. It is also worthwhile noting that the spatial part of the wave operator can be factorized as $\boldsymbol{B}^{\dagger} \boldsymbol{B}$, with $\boldsymbol{B}$ regular. This implies that the Schwarzschild solution is linearly stable in the odd-parity sector. It turns out that this factorization can be generalized in the presence of a SU(2) Yang-Mills field. This then implies the absence of non-spherically symmetric unstable odd-parity modes for the Bartnik-McKinnon solitons 14 and the corresponding black holes with hair [15] [13.

\section{The even-parity sector}

In the even-parity sector $L_{i j}$ is expanded according to 


$$
\begin{aligned}
& L_{x x}=n_{1} \frac{h}{r} Y, \\
& L_{x B}=n_{2} q \hat{\nabla}_{B} Y, \\
& L_{A B}=-n_{3} h e_{A B}^{(3)}+n_{4} g e_{A B}^{(4)},
\end{aligned}
$$

where

$$
e_{A B}^{(3)}=r \hat{g}_{A B} Y, \quad e_{A B}^{(4)}=r\left(\hat{\nabla}_{A} \hat{\nabla}_{B} Y+\frac{1}{2} \ell(\ell+1) \hat{g}_{A B} Y\right) \text {. }
$$

The basis is chosen such that $L_{i j}$ is trace-less and orthonormal with respect to the inner product (24):

$$
\left\langle L_{i j}, L_{i j}\right\rangle=\int_{0}^{\infty}\left(h^{2}+k^{2}+g^{2}\right) d x .
$$

One finds

$$
n_{1}=\sqrt{\frac{2}{3}}, \quad n_{2}=\frac{1}{\sqrt{2 \mu^{2}}}, \quad n_{3}=\frac{1}{\sqrt{6}}, \quad n_{4}=\sqrt{\frac{2}{\mu^{2} \lambda}},
$$

where we have defined $\mu^{2}=\ell(\ell+1)$ and $\lambda=(\ell-1)(\ell+2)$.

In contrast to the odd-parity case, the amplitudes $h, q$ and $g$ are subject to residual coordinate transformations of the form (16). Expanding $f=\xi(x) Y$, we find

$$
\begin{aligned}
& n_{3} h \mapsto n_{3} h-\alpha^{2} r^{\prime} \xi^{\prime}+\frac{\mu^{2}}{2} \frac{\alpha^{2}}{r} \xi, \\
& n_{2} q \mapsto n_{2} q+\alpha r\left(\frac{\alpha}{r} \xi\right)^{\prime}, \\
& n_{4} g \mapsto n_{4} g+\frac{\alpha^{2}}{r} \xi,
\end{aligned}
$$

where $f$ obeys the equation $\bar{\nabla}^{k}\left(\alpha^{2} \bar{\nabla}_{k} f\right)=0$, i.e.

$$
-\frac{1}{\alpha^{2} r^{2}}\left(\alpha^{2} r^{2} \xi^{\prime}\right)^{\prime}+\frac{\mu^{2}}{r^{2}} \xi=0 .
$$

As the dynamical variables must be coordinate-invariant, these residual transformations will help us to construct them as appropriate combinations of the amplitudes $(h, g, q)$. Using (A4) in $\hat{\Lambda}_{i j}=0$ yields the wave equation

$$
\left(\partial_{t}^{2}-\partial_{\rho}^{2}+\boldsymbol{S}\right) u+\boldsymbol{b}(a)=0,
$$

where the symmetric matrix $\boldsymbol{S}$ is given by

$$
\boldsymbol{S}=\left(\begin{array}{ccc}
\gamma^{2} S_{11} & \sqrt{12} \mu \gamma_{\rho} & 0 \\
\sqrt{12} \mu \gamma_{\rho} & \gamma^{2} S_{22} & 2 \sqrt{\lambda} \gamma_{\rho} \\
0 & 2 \sqrt{\lambda} \gamma_{\rho} & \gamma^{2} S_{33}
\end{array}\right)
$$

with

$$
\begin{aligned}
& S_{11}=\mu^{2}+6 r^{2}-5 r r^{\prime \prime}-9 r r^{\prime} \frac{\alpha^{\prime}}{\alpha} \\
& S_{22}=\mu^{2}+4 r^{\prime 2}-4 r r^{\prime \prime}-8 r r^{\prime} \frac{\alpha^{\prime}}{\alpha}+\frac{r^{2}}{\alpha^{2}}\left(\alpha \alpha^{\prime}\right)^{\prime}, \\
& S_{33}=\mu^{2}-2 r^{\prime 2}-r r^{\prime \prime}-r r^{\prime} \frac{\alpha^{\prime}}{\alpha} .
\end{aligned}
$$

The inhomogeneous term $\boldsymbol{b}(a)$, where $a=a(x)$ is the scalar amplitude parametrizing $A, A=\alpha a Y$, is found to be

$$
\begin{aligned}
& n_{3} b_{1}=2 \alpha^{2} r^{\prime} a^{\prime}-\mu^{2} \frac{\alpha^{2}}{r} a \\
& n_{2} b_{2}=-2 \alpha r\left(\frac{\alpha}{r} a\right)^{\prime}, \\
& n_{4} b_{3}=-\frac{2 \alpha^{2}}{r} a .
\end{aligned}
$$

Next, the $\Lambda_{k}^{k}$-equation yields

$$
\frac{2}{r}\left[\left(r^{2}(\alpha a)^{\prime}\right)^{\prime}-\alpha \mu^{2} a\right]+\frac{4 n_{3}}{\alpha}\left[\alpha \alpha^{\prime} \frac{r^{\prime}}{r}-\left(\alpha \alpha^{\prime}\right)^{\prime}\right] h=0 .
$$

This equation has already been used in our derivation in order to eliminate the second derivatives of $a$ in the expression for $b_{1}$. Finally, the linearized momentum constraint yields the following two equations:

$$
\begin{aligned}
& 0=C_{x}=2 n_{3}\left[\frac{\alpha}{r^{2}}\left(\frac{r^{2} h}{\alpha}\right)_{\rho}-\frac{\sqrt{3} \mu}{2} \gamma q\right] \frac{Y}{r}, \\
& 0=C_{B}=n_{2}\left[\frac{\alpha}{r^{2}}\left(\frac{r^{2} q}{\alpha}\right)_{\rho}-\frac{\sqrt{3} \mu}{3} \gamma h-\sqrt{\lambda} \gamma g\right] \hat{\nabla}_{B} Y .
\end{aligned}
$$

Unfortunately, we did not succeed in finding a transformation from the original variables $u$ to the dynamical variables $u_{p}$ in a similarly elegant way like in the oddparity sector, i.e. by means of a supersymmetry transformation. However, the dynamical degrees of freedom are found systematically as follows: First, one has three original variables $u=(h, q, g)$ and two constraint variables, which are given by Eqs. (A13). We are, therefore, looking for a single dynamical scalar amplitude. This amplitude must be invariant with respect to the residual gauge transformation (A9). The simplest way to construct a completely coordinate-invariant amplitude is to take a linear combination of the amplitudes $h, q$ and $g$. Requiring invariance with respect to the residual transformation (A9), the only possibility (up to rescaling) is

$$
Z \equiv n_{3} h+n_{2} r^{\prime} q-n_{4} f g,
$$

where the function $f=f(x)$ is given by

$$
f(x)=\frac{1}{2} \mu^{2}+r r^{\prime} \frac{\alpha^{\prime}}{\alpha}-r^{\prime 2} .
$$

Taking the corresponding combination of the evolution equations (A10) yields

$$
\left(\partial_{t}^{2}-\partial_{\rho}^{2}\right) Z+\gamma^{2} \mu^{2} Z-6 \alpha \alpha^{\prime} \frac{r^{\prime}}{r} Z-6 n_{2} \alpha \alpha^{\prime} \frac{r^{\prime}}{r} \hat{q}=0,
$$

where the background equations ( $\mathrm{A3}$ ) have been used in order to simplify the expression. The coordinateinvariant amplitude $\hat{q}$ is given by 


$$
\hat{q}=q-r \frac{n_{4}}{n_{2}}\left(\frac{g}{\alpha}\right)_{\rho} .
$$

Note that the lapse amplitude $a$ does not appear in the above equation. This becomes clear when one compares (A12) with (A9): Any gauge-invariant combination of the evolution equations annihilates the terms involving a. The constraint equations, (A13) yield

$$
\left(\frac{Z}{\gamma}\right)_{\rho}+n_{2} f \hat{q}=0
$$

The amplitude $\hat{q}$ can therefore be eliminated. After using the background expressions

$$
\alpha^{2}=r^{\prime 2} \equiv N=1-\frac{2 G m}{r}, \quad \alpha \alpha^{\prime} \frac{r^{\prime}}{r}=\frac{G m N}{r^{3}},
$$

we obtain the scalar equation

$$
\left(\lambda+\frac{6 G m}{r}\right)\left(\partial_{t}^{2}-\partial_{\rho}^{2}\right) Z-\frac{12 G m}{r^{2}} N Z_{\rho}+\mu^{2} \lambda \frac{N}{r^{2}} Z=0 .
$$

Defining $Z=(\lambda+6 G m / r) \Psi$, this is the Zerilli equation [9].

Finally, we mention that the separation between the constraint and dynamical variables can also be achieved by a first order transformation of the form $\Psi=A h+$ $B q+C g+g_{\rho}$ with suitable functions $A, B$ and $C$. This ansatz results in the Regge-Wheeler equation [20]. A similar result has been obtained in [6], however, in contrast to their derivation, the procedure adopted here is more natural, since the requirement of gauge invariance with respect to reparametrization of the time coordinate implies that only one function must be matched.

\section{APPENDIX B: PERFECT FLUIDS AS FIELD THEORY}

In this appendix, we briefly review a field theoretical formulation of perfect fluids models [21] and discuss some applications to linear perturbation theory.

The fluid's motion is described by a map

$$
F: M \rightarrow \Gamma, x \mapsto\left(F_{1}(x), F_{2}(x), F_{3}(x)\right),
$$

where $(M, \boldsymbol{g})$ denotes space-time and where $\Gamma$ is a threedimensional manifold. The fields $F_{1}, F_{2}$ and $F_{3}$ are scalar fields on $M$ and describe a material coordinate system for the fluid. We assume that the linear map $F_{* x}: T_{x} M \rightarrow$ $T_{F(x)} \Gamma$ has rank three and that the kernel of $F_{* x}$ is timelike for all points $x \in M$.

Let $\Omega=\bar{n}(F) d F_{1} \wedge d F_{2} \wedge d F_{3}$ be a volume form on $\Gamma$, where $\bar{n}(F)$ describes the material density. Then, we define a 4 -current $J$ by

$$
J \equiv *\left(F^{*} \Omega\right)
$$

where $F^{*}$ is the pull-back and $*$ the Hodge-dual on $(M, \boldsymbol{g})$. In local coordinates, we have

$$
J_{\mu}=\bar{n}(F) \eta_{\mu \alpha \beta \gamma}\left(\nabla^{\alpha} F_{1}\right)\left(\nabla^{\beta} F_{2}\right)\left(\nabla^{\gamma} F_{3}\right)
$$

[As an example, consider $F_{a}\left(t, x^{a}\right)=x^{a}-t v^{a}, v^{a}=$ const. which describes a fluid in constant motion in flat space. Then we have $J^{t}=\bar{n}, J^{i}=\bar{n} v^{i}$.] The definition of $J$ implies that

$$
* d * J=0,
$$

thus the particle number is conserved. Note also that $J$ spans the kernel of $F_{*}$. The particle density and the 4 -velocity, as measured in the coordinate system $x$, are defined by

$$
n=\sqrt{-\boldsymbol{g}(J, J)}, \quad \text { and } \quad u^{\mu}=\frac{1}{n} J^{\mu},
$$

respectively.

The dynamics of the fluid is described by a Lagrangian of the form

$$
\mathcal{L}\left(F_{a}, \nabla F_{a}\right)=-n u(v) .
$$

Here, $u$ is a function of $v=1 / n$ and describes the inner energy per particle. Some calculations show that the stress-energy tensor, $T^{\mu \nu}=2 \frac{\delta \mathcal{L}}{\delta g_{\mu \nu}}+g^{\mu \nu} \mathcal{L}$, is given by

$$
T^{\mu \nu}=(\rho+P) u^{\mu} u^{\nu}+g^{\mu \nu} P,
$$

where $\rho=n u$ is the energy density and

$$
P=-\frac{\partial u}{\partial v}
$$

is the pressure. Note that this agrees with the first law of thermodynamics, $\delta u=-P \delta v$, when the entropy per particle is constant. Using $T^{\mu \nu}=g^{\mu \nu} \mathcal{L}-\left(\partial^{\mu} F_{a}\right) \frac{\partial \mathcal{L}}{\partial\left(\partial_{\nu} F_{a}\right)}$, one also finds

$$
\nabla_{\nu} T_{\mu}^{\nu}=\left(\partial_{\mu} F_{a}\right)\left[\frac{\partial \mathcal{L}}{\partial F_{a}}-\nabla_{\nu} \frac{\partial \mathcal{L}}{\partial\left(\partial_{\nu} F_{a}\right)}\right] .
$$

Hence, the relativistic Euler equations, $\nabla_{\nu} T_{\mu}^{\nu}=0$, are equivalent to the Euler-Lagrange equations with respect to (B1), since $F_{*}$ was required to have full rank.

Consider linear perturbations of a self-gravitating fluid, described by the (Eulerian) perturbations $\delta g_{\mu \nu}$ and $\delta R_{a}$. One can also introduce the Lagrangian perturbation operator $\Delta=\delta+L_{\xi}$, where the Lagrangian displacement field $\xi$ is a first order quantity defined such that the material coordinates do not change under the perturbations:

$$
\Delta F_{a}=0, \quad a=1,2,3 .
$$

Since $F_{a}$ are scalar fields, this means that

$$
\delta F_{a}=-\left(\partial_{\mu} F_{a}\right) \xi^{\mu}
$$

Therefore, $\xi^{\mu}$ is uniquely defined up to a vector proportional to $u^{\mu}$. Furthermore, under an infinitesimal coordinate transformation generated by a vector field $X^{\mu}$, we must have 


$$
\xi^{\mu} \mapsto \xi^{\mu}-X^{\mu}
$$

(modulo a vector proportional to $u^{\mu}$ ) in order to maintain equation (B3). As an important consequence of (B4), Lagrangian perturbations of a tensor quantity are automatically coordinate invariant to linear order.

Since $\Delta R_{a}=0$ and $[d, \Delta]=0, \Delta J^{\mu}$ and therefore also $\Delta n, \Delta u^{\mu}, \Delta \rho$ and $\Delta P$ can be expressed in terms of $\Delta \boldsymbol{g}$ only. Using

$$
\Delta J_{\mu}=-\frac{1}{2}\left(g^{\alpha \beta} \Delta g_{\alpha \beta}\right) J_{\mu}+J^{\alpha} \Delta g_{\alpha \mu},
$$

the following relations can be derived in quite an efficient way

$$
\begin{aligned}
\Delta n & =-\frac{1}{2} n q^{\alpha \beta} \Delta g_{\alpha \beta}, \\
\Delta u^{\mu} & =\frac{1}{2}\left(u^{\alpha} u^{\beta} \Delta g_{\alpha \beta}\right) u^{\mu}, \\
\Delta \rho & =-\frac{1}{2}(\rho+P) q^{\alpha \beta} \Delta g_{\alpha \beta}, \\
\Delta P & =-\frac{1}{2} \gamma P q^{\alpha \beta} \Delta g_{\alpha \beta} .
\end{aligned}
$$

Here, we have also defined the adiabatic index

$$
\gamma=\frac{\partial \log P}{\partial \log n}=\frac{n^{3}}{P} \frac{\partial^{2} u}{\partial v^{2}},
$$

and $q^{\alpha \beta}=g^{\alpha \beta}+u^{\alpha} u^{\beta}$. These relations, which are wellknown (see, e.g., 222), imply that the Lagrangian perturbations of the particle conservation equation and the continuity equation are fulfilled. More precisely, we have

$$
\begin{aligned}
\frac{\Delta}{n}\left(\nabla_{u} n+n \theta\right) & =\nabla_{u}\left(\frac{\Delta n}{n}+\frac{1}{2} q^{\alpha \beta} \Delta g_{\alpha \beta}\right), \\
\frac{\Delta}{\rho+P}\left(\nabla_{u} \rho+(\rho+P) \theta\right) & =\nabla_{u}\left(\frac{\Delta \rho}{\rho+P}+\frac{1}{2} q^{\alpha \beta} \Delta g_{\alpha \beta}\right),
\end{aligned}
$$

where $\theta=\nabla_{\mu} u^{\mu}$.

[1] R.H. Price and J. Pullin, Phys. Rev. Lett. 72, 3297 (1994).

[2] O. Brodbeck, M. Heusler, N. Straumann, and M. Volkov, Phys. Rev. Lett. 79, 4310 (1997).

[3] O. Sarbach, M. Heusler, and O. Brodbeck, Perturbation theory for self-gravitating gauge fields The odd-parity sector, Phys. Rev. D62, 084001 (2000).

[4] Perturbation theory for self-gravitating gauge fields The even-parity sector (in preparation).

[5] Y. Choquet-Bruhat, J.W. York, Jr, and A. Anderson, Curvature-based hyperbolic systems for general relativity, gr-qc/9802027; A. Abrahams, A. Anderson, Y. ChoquetBruhat, and J.W. York, Jr. , Phys. Rev. Lett. 75, 3377 (1995).
[6] A. Anderson, A.M. Abrahams, and C. Lea, Phys. Rev. D58, 064015 (1998).

[7] O. Brodbeck, M. Heusler, and N. Straumann, Phys. Rev. D53, 754 (1996).

[8] T. Regge and J. Wheeler, Phys. Rev. 108, 1063 (1957).

[9] F.J. Zerilli, Phys. Rev. Lett. 24, 737 (1970).

[10] V. Moncrief, Phys. Rev. D9, 2707 (1974); Phys. Rev. D10, 1057 (1974); Phys. Rev. D12, 1526 (1975).

[11] O. Brodbeck, M. Heusler, and O. Sarbach, Phys. Rev. Lett. 84, 3033 (2000).

[12] J. W. York Jr., J. Math. Phys. 14, 456 (1973).

[13] O. Sarbach (in preparation)

[14] R. Bartnik and J. McKinnon, Phys. Rev. Lett. 61, 141 (1988).

[15] M.S. Volkov and D.V. Gal'tsov, JETP Lett. 50, 346 (1989); H.P. Künzle and A.K.M. Masood-ul-Alam, J. Math. Phys. 31, 928 (1990); P. Bizon, Phys. Rev. Lett. 64, 2844 (1990).

[16] E. Winstanley, Class, Quantum Grav. 16, 1963 (1999).

[17] B. Kleihaus and J. Kunz, Phys. Rev. Lett. 79, 1595 (1997).

[18] V. Moncrief, Ann. Phys. 88, 343 (1974).

[19] G. Allen, N. Andersson, K.D. Kokkotas, and B.F. Schutz, Phys. Rev. D 58, 124012 (1998).

[20] U. Straumann, On the Regge-Wheeler perturbation equations, Diploma thesis, University of Zurich (unpublished).

[21] D. E. Soper, Classical Field Theory, John Wiley \& Sons (1976).

[22] J. L. Friedman and J. R. Ipser, Phil. Trans. R. Soc. Lond. A 340, 391 (1992). 